\title{
Music of the \\ Minnesinger and \\ Early Meistersinger
}

\section{A Bibliography}


Music of the Minnesinger and Early Meistersinger 


\section{IIIIUNC}

From 1949 to 2004, UNC Press and the UNC Department of Germanic \& Slavic Languages and Literatures published the UNC Studies in the Germanic Languages and Literatures series. Monographs, anthologies, and critical editions in the series covered an array of topics including medieval and modern literature, theater, linguistics, philology, onomastics, and the history of ideas. Through the generous support of the National Endowment for the Humanities and the Andrew W. Mellon Foundation, books in the series have been reissued in new paperback and open access digital editions. For a complete list of books visit www.uncpress.org. 



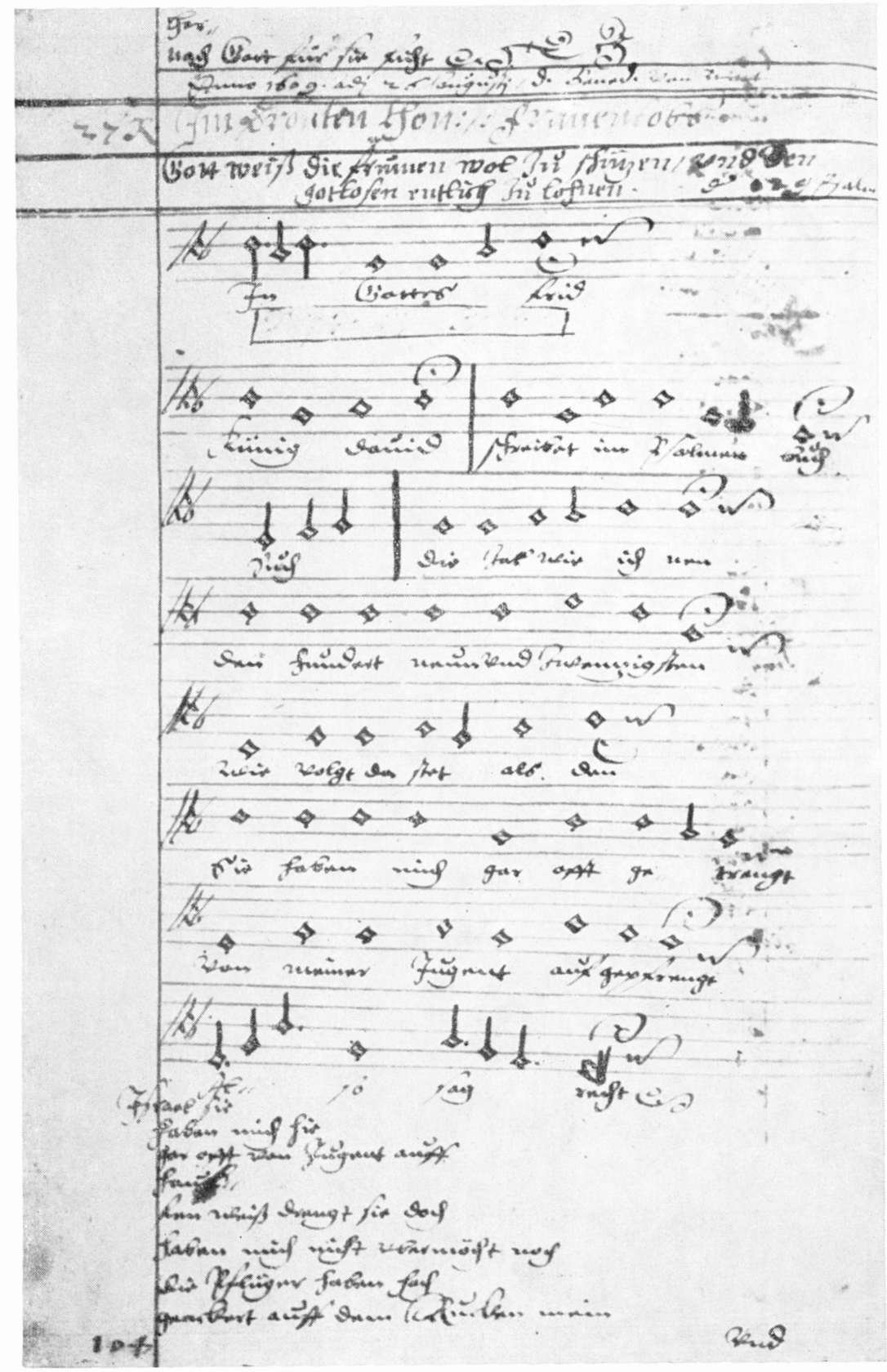

Berlin, Germ. Fol, 24, f. 224v. 


\section{Music of the Minnesinger and}

Early Meistersinger

A Bibliography

ROBERT WHITE LINKER

UNC Studies in the Germanic Languages and Literatures

Number 32 
Copyright (C) 1962

This work is licensed under a Creative Commons CC BY-NC-ND license. To view a copy of the license, visit http://creativecommons. org/licenses.

Suggested citation: Linker, Robert White. Music of the Minnesinger and Early Meistersinger: A Bibliography. Chapel Hill: University of North Carolina Press, 1962. DOI: https://doi.org/ 10.5149/9781469657806_ Linker

Library of Congress Cataloging-in-Publication Data Names: Linker, Robert White.

Title: Music of the Minnesinger and Early Meistersinger : A bibliography / by Robert White Linker.

Other titles: University of North Carolina Studies in the Germanic Languages and Literatures; no. 32.

Description: Chapel Hill : University of North Carolina Press, [1962] Series: University of North Carolina Studies in the Germanic Languages and Literatures. | Includes bibliographical references. Identifiers: LCCN 62062958 | ISBN 978-0-8078-8832-2 (pbk: alk. paper) | ISBN 978-1-4696-5780-6 (ebook)

Subjects: Songs, German — Bibliography. | Minnesingers. | Meistersinger.

Classification: LCC PD25 .N6 NO. 32 | DCC 016/.7800712 


TO

FOUR GENERATIONS OF

GOOD FRIENDS

GENE, BOYKIN, LARRIE, EUGENE 



\section{PREFACE}

The study of the songs of the Minnesinger is complicated by the fact that most of the tunes have come down to us only in late manuscripts, with the original Ton or Weise accompanied by new texts by later poets, leaving questions of authenticity or corruption to be solved. In addition to this, histories of mediaeval music frequently treat as Minnesinger certain poets whom the historian of literature considers as Meistersinger. I have not attempted to settle this disagreement, but have included both groups, with dates, wherever available, to distinguish between the earlier Minnesinger and the later Meistersinger.

The bibliography is arranged alphabetically by composer, with each of his songs in alphabetical order following any general work on the composer. It will be noted that the same tune may occur a number of times, with different words. Perhaps I should have eliminated this multiple use, but since many of these entries have become part of musical literature, I have presented them as found. Under each song is listed, where pertinent and available to me, the manuscript and folio, any ascription or designation of tune contained in the manuscript, publication, with reproduction of mediaeval notation designated by $\%$, transcription into modern notation by $\mathrm{d}$. Occasionally I have cited editions of the text without music.

The list of manuscripts at the beginning of the book gives general facts on the manuscript, and any publication or facsimile reproduction. Thereafter the manuscripts are cited by place and number, or the more extensive manuscripts such as the Colmar or Jena by name. After the list of manuscripts is given a list of publications with the abbreviations used for them.

There are doubtless many shortcomings to this bibliography, chief among which is the need for re-assignment to the original words of tunes borrowed by later writers, yet I hope that an orderly listing will be of use to students of German literature and music.

No collection of material such as this would have been possible without the help of my friends and the libraries I have secured materials from. Among the many who have helped, I should like especially to thank Professor John G. Kunstmann for the use of his books and his knowledge, Miss Thelma Thompson and Mr. Keith Mixter for numerous friendly aids, Dr. Ewald Jammers of Heidelberg, Dr. Wilhelm Virneisel of Tübingen, for their very generous checking and securing for me basic materials. Lastly, I am very grateful to my fellow alumni, whose financial assistance, through the University Research Council, have made publication possible. 



\section{MANUSCRIPTS}

The greatest of the manuscripts of the poetry of the Minnesinger is the Manesse, or Grosse Heidelberger: Heidelberg, Universitätsbibliothek, Cod. Pal. Germ. 848. Copiously illustrated with Minnesinger portraits and scenes, but without music. It has been reproduced in facsimile by the Inselverlag, Leipzig, I925-29.

\section{MUSIC}

I. Bartsch's fragment: See 60-5.

2. Basel, Universitätsbibliothek, Fragmentenband, N.J. 3, nr. I45. See I7 (Meister Kelin), 53 (Vegeviur), and JHSB II, I 54.

3. Berlin, Preussische Staatsbibliothek, Ms. germ. fol. 24. Paper, xvi c. Meisterlieder, including Minnesinger tunes. $277 \mathrm{ff}$.

Fol. 223 begins old series of numbers as f.r. See HM IV, 907, nos. 6467; also non-musical manuscripts below. (Now in Tübingen: see s below.

4. Berlin, Preussische Staatsbibliothek, Ms. germ. fol. 25 (Now in Tübingen, see s below). Xvi C. 460 pp. See HM IV, 907, nos. 64-7.

5. Berlin, Preussische Staatsbibliothek, Ms. germ. fol. 779,ff. I 3 I-269. (Now Tübingen, Universitätsbibliothek, Depot der ehem. Preuss. Staatsbibliothek. Ms. germ. fol. 779) Friedrich von der Hagen's Neidhart manuscript. Facsimile: W. Schmieder, DTO LXXI (I930), 3-20, 3I-39. See further: Keinz, Beiträge zur Neidhartforschung, Bayr.Sitz.Ber., Phil.-hist. Kl. (I888), II, 3 I 2. Brill, Die Scbule Neidharts, 45 ff. Haupt-Wiessner, Neidhart von Reuenthal, viII ff.

6. Berlin, Preussische Staatsbibliothek, Ms. germ.fol. 922. xv c. Music, ff. 13 1-34. (Now Tübingen, Universitätsbibliothek: see 5 above). Margarete Lang, Zwischen Minnesang und Volkslied. Die Lieder der Berliner Hs. germ. fol. 922. Die Weisen bearbeitet von Joseph M. Müller-Blattau (Studien zur Volksliedforschung, hrsg. von John Meier, Heft I. Berlin, I94I). Carl von Kraus, $Z u$ den Liedern der Berliner Hs. germ. fol. 922 , / Abhandlungen der Bayer. Akad. d. Wissenschaft, Phil.hist. Abtlg., N.F., Heft 21, München, 1942.

7. Berlin, Preussische Staatsbibliothek, Ms. germ. $4^{\circ}$, 981. Now Westdeutsche Bibliothek, Marburg). One folio, parchment, from Magdeburger Archiv. Facsimile, HM IV, 773; Wolf, Handbuch der Notationskunde.

See 60-2.

- Breslau: See Wrocław.

- Colmar: See München. 
8. Donaueschingen, Fürstlich-Fürstenbergische Bibliothek, Hs. I20. XV-Xvi C.

Runge, Die Sangesweisen der Colmarer Handscbrift und Liederbandschrift Donauescbingen. Leipzig, Breitkopf und Härtel, I 896.

Karl Bartsch, Meisterlieder, 89ff., gives a table of contents.

9. Engelberg Ms. See 26-I.

ıо. Erlangen, Universitätsbibliothek, Ms. 1655. xv c. Paper. I4 ff. See HM IV, 904a.

I I. Frankfurt-am-Main, Staatsbibliothek, Neidhart fragment, 2 ff. See HM IV, 770-7I. Facsimile, DTO LXXI.

I 2. Heidelberg, Universitätsbibliothek, Hs. 329. See is (Hugo von Montfort).

I 3. Innsbruck, Universitätsbibliothek. See 3 I (Oswald von Wolkenstein).

I4. Jena, Universitätsbibliothek, Jenaer Liederhandschrift. Facsimile: K. K. Müller, Die Jenaer Liederbandscbrift in Licbtdruck. Jena, I 896. Holz, Saran, Bernoulli, Die Jenaer Liederbandschrift, Leipzig, Hirschfeld, I901. 2 vols. Karl Bartsch, Untersuchung zur Jenaer Liederbandschrift (Palaestra 140). Leipzig, I923. Karl B. Brandis, „Zur Entstehung und Geschichte der Jenaer Liederhandschrift", Z.f. Bücherfreunde XxI, 108. E. Jammers, „Untersuchungen über die Rhytmik und Melodik der Melodien der Jenaer Liederhandschrift", zf MW VII (I924-25), 265-304. HM IV, reproduction of original notation.

- Kolmar: See München.

I 5. Königsberg, Univ. Bibl. 7 ff. See 8-7.

16. Kremsmünster, Stiftsbibliothek, Ms. I 27, vII, I8, f. I 30 ab. See Franz Pfeiffer, Germania II (I 857), 470.

17. Leipzig, Universitätsbibliothek, ms. I305, ff. 107-I 10. See I9 (Konrad von Queinfurt).

- Magdeburg, Archiv: See Berlin 981.

Facsimile: HM IV, 773

I 8. München, Staatsbibliothek, Cod. germ. 4997. Paper. xv c. The Colmar Manuscript.

Paul Runge, Die Sangesweisen der Colmarer Handscbrift und die Liederbandschrift Donauescbingen. Leipzig, Breitkopf und Härtel, I 896. Friedrich Ebert, Die Liedweisen der Kolmarer Handscbrift und ibre Einordnung und Stellung in der Entwicklungsgescbichte der deutschen Liedweise im 14. bis I6. Jabrbundert. I I4 pp. Diss. Göttingen, I933. Rudolf Zitzmann, Die Melodien der Kolmarer Liederbandschrift. Würzburg, Triltsch, I944. Karl Bartsch, Meisterlieder der Kolmarer Handschrift. Stuttgart, Bibliothek des litterarischen Vereins in Stuttgart, LVIII, I 862. Heinz Otto Burger, Die Kunstauffassung der früben Meistersinger, ein Untersuchung über die Kolmarer Handschrift. Berlin, Junker und Dünnhaupt, 1936 . $80 \mathrm{pp}$. 
I9. München, Staatsbibliothek, Cgm. 5249/32. Fragment. See 8-7. München fragment M: Facsimile in Eduard Bernoulli, Die Choralnotenscbrift bei Hymnen und Sequenzen. Leipzig, Breitkopf u. Härtel, 1898.

20. München, Staatsbibliothek, Clm. 5509. Lost. See KLD, pp. XXI-XXII.

21. München, Staatsbibliothek, Clm. 5530 .

22. München, Staatsbibliothek, Clm. 5539 . See 49-4.

23. München, Docen, fragment of Walther von der Vogelweide. See HM IV, 90I, no. Io Lost.

24. München, Staatsbibliothek, Cod. Lat. 4660 (Carmina Burana). See KLD, p. XIX; also 30-I6.

25. Münster, Staatsarchiv, Ms. vII, 5 I. Fragment, 4 pp., five songs, only one complete. XIV-XV C.

R. F. Molitor, „Die Lieder des Münsterischen Fragmentes,” sIMG XII (I I IO-I I), 475-500, bibliography, 475, facsimile, 5ooff. F. Jostes, „Bruchstück einer Münsterschen Minnesängerhandschrift, mit Noten", zfdA LIII (I9I 2), 348-57.

26. Nürnberg, Stadtbibliothek, Ms. 784: see 57-6.

27. Nürnberg, Stadtbibliothek, Ms. 792: see 57-6.

28. Schreiber fragment. Lost.

H. Schreiber, Taschenbuch $f$. Geschichte und Altertum in Süddeutschland, I 838; HM IV, 772; Hugo Kuhn, Minnesangs Wende, Tübingen, I952. Facsimile in all three.

See 50-I.

- Sterzing: See Vipiteno.

29. Stettin, Schulbibliothek. Fragment.

See HM IV, 904, no. 39; нм IV, 769, Tafel v.

30. Strassburg.

See 32-2.

31. Trier, Stadtbibliothek, Hs. 724. XV C. See 32-2.

32. Vipiteno, Stadtarchiv. See 30 (Neidhart von Reuenthal) and H. Rietsch, Die deutsche Liedweise (1904), p. 2 I 5 f. Facsimile, DTO LXXI.

33. Wien, Nationalbibliothek, Hs. 270r. Paper. xiv c.

HM IV, $768,769,774$. H. Rietsch, DTO XLI, facsimile and transcription. G. Roethe, Reinmar von Zweter, I47f.

34. Wien, Nationalbibliothek, Hs. 2777. See 3 I (Oswald von Wolkenstein) and DTO XviIr.

35. Wien, Nationalbibliothek, Hs. 2856 (Mondseer Hs.). See I 3-4, I 3-10 and Germanica III-IV.

36. Wien, Nationalbibliothek, ms. suppl. 3344. See 30 (Neidhart von Reuenthal), and DTO LXxi. Facsimile, DTO LXxi. 
37. Wien, Nationalbibliothek, Titurel Hs. 40 (Ambr. 42I). HM IV, 774; facsimile, HM IV, 903 , no. 30 .

38. Wien, K. K. Fideicommissbibliothek, Cod. membr. 7970. See 32-2.

39. Wrocław, Biblioteka Universytecka, Ms. 356. Adam Puschman's Singebuch. Reported lost during World War II.

See partial publication below, under APSB.

40. Wrocław, Bibl. Univ., Ms I.Q. 368.

See 8-7, and Paul Pietsch, ZfdP xIv (1882), 98-99.

\section{NON-MUSICAL MANUSCRIPTS}

Frequently mentioned in connection with the Minnesinger are, among others, the following manuscripts containing poetic texts, but without notation of music:

Berlin, Staatsbibliothek, Hss. germ. $f^{\circ}, 22$ and 23. (Now in Tübingen). See s above.

Heidelberg, Universitätsbibliothek, Hss. I09, 392, and 680.

\section{General:}

PUBLICATIONS

I. Archer Taylor, The Literary History of Meistergesang. New York, Modern Language Association of America, 1937.

2. Karl Goedeke, Grundriss zur Gescbicbte der deutschen Dicbtung aus den Quellen. Dresden, Ehlermann, I884.

3. Die deutsche Literatur des Mittelalters. Verfasser Lexikon. Vols. I-II, ed. Wolfgang Stammler; III-v, ed. Karl Langosch. Berlin, Walter de Gruyter \& Co.

4. TEBM: Archer Taylor and Frances $\mathrm{H}$. Ellis, A Bibliograpby of Meistergesang. Indiana University Studies, xxIIr, 1936. Pp. 92.

Music and Texts:

5. Ursula Aarburg, „Melodien zum frühen deutschen Minnesang," zfdA LXXXVII (1956-57), 24-45.

6. AfMw: Archiv für Musikwissenschaft.

7. ApsB: G. Münzer, Das Singebuch des Adam Puschman nebst den Originalmelodien. Leipzig, Breitkopf und Härtel, I 906, 96 pp. Melodies published, in modern notation, indicated below by

8. вмкн: Karl Bartsch, Meisterlieder der Kolmarer Handschrift. Bibliothek des litterarischen Vereins in Stuttgart, Lxvirr, Stuttgart, 1862. 
9. DAHA: Archibald T. Davison and Willi Apel, Historical Anthology of Music, Vol. I. Harvard University Press, I947.

Iо. DTO: Denkmäler der Tonkunst in Österreich. DTO XLI: Gesänge von Frauenlob, Reinmar v. Zweter und Alexander, nebst einem anonymen Brucbstück nach der Handschrift 2701 der Wiener Hofbibliothek bearbeitet von Heinrich Rietsch mit Reproduction der Handschrift. Wien, Artaria, I9I 3.

DTO XVIII: see Oswald von Wolkenstein.

DTO LXXI: see Neidhart von Reuenthal.

II. GFML: Friedrich Gennrich, Grundriss einer Formelebre des mittelalterl. Liedes. Halle, Niemeyer, I 932.

I2. Fr. Gennrich, „Liedkontrafactur in mhd. und ahd. Zeit,” zfdA LXXXII (I948), I05-4I.

I3. Fr. Gennrich, „Mittelalterliche Lieder mit textloser Melodie,” AfMw Ix (I952), I $20 f f$.

14. Friedrich Gennrich, Melodien altdeutscher Lieder. Darmstadt, I954.

I5. Fr. Gennrich, Mittelbochdeutsche Liedkunst. Darmstadt, I954.

16. Fr. Gennrich, Übertragungsmaterial zur Rhytbmik der Ars Antiqua. Darmstadt, I954.

17. GMMA: Théodor Gérold, La Musique au moyen âge. Paris, Cfmâ, Champion, 1932.

18. GTM: Friedrich Gennrich, Troubadours, Trowvères, Minne- und Meistergesang. Köln, Volk, I95 I, 1960.

19. HGEM: Harold Gleason, Examples of Music before 1400. New York, Crofts, 1946. II7 pp.

20. HM: Friedrich von der Hagen, Minnesinger. 5 vols. Leipzig, Barth, I 838. Vol. IV contains bibliography of Minnesinger manuscripts, facsimiles, and reproduction of original notation of Jena manuscript (pp. 775844 ), and of the songs of Neidhart (pp. 845-8 2 2).

21. JHSB: Holz, Saran, Bernoulli, Die Jenaer Liederbandscbrift. 2 vols. Leipzig, Hirschfeld, r90r.

22. KLD: Carl von Kraus, Deutsche Liederdicbter des 13. Jabrbunderts. Tübingen, Niemeyer, 1952.

23. Kuhn-Reichert: Hugo Kuhn, Minnesang des 13. Jabrbunderts... mit Übertragung der Melodien von Georg Reichert. Tübingen, Niemeyer, I953. Pp. I60.

24. Lang-Salmen: Margarete Lang, Ostdeutscher Minnesang...

Melodien hrsg. v. Walter Salmen. Lindau, Kostanz, Thorbecke, 1958.

25. MGDM: Hans Joachim Moser, Geschichte der deutschen Musik. Stuttgart, Berlin, Cotta, 1930.

26. Nонм: The New Oxford History of Music. Vol. II, Early Medieval Music up to 1300. Oxford University Press, I954.

27. PC: Alfred Pillet, Henry Carstens, Bibliographie der Troubadours. Halle, Niemeyer, 1933. 
28. PMDL: Fridrich Pfaff, Der Minnesang des I2. bis I4. Jabrbunderts. (Deutsche National-Literatur, VIII). Stuttgart, Union, ca. I 890.

29. Riemann: Hugo Riemann, Handbuch der Musikgescbicbte, I, 2, Die Musik des Mittelalters. Leipzig, Breitkopf und Härtel, I905.

30. Rmma: Gustave Reese, Music in the Middle Ages. New York, Norton, I940.

31. SGMB: Arnold Schering, Geschichte der Musik in Beispielen. Leipzig, Breitkopf und Härtel, I93 I.

32. SIMG: Sammelbände der Internationalen Musikgesellschaft.

33. Das Taghorn. Dichtungen und Melodien des Bayrisch-Oesterreichischen Minnesanges, ed. Alfred Rottauscher und B. Paumgartner. 3 vols. Wien, I922.

34. Ronald J. Taylor, „Zur Übertragung der Melodien der Minnesänger,” zfda Lxxxvir (1956-57), 1 32-47.

35. Otto Ursprung, "Vier Studien zur Geschichte des deutschen Liedes," I. „Mein traut gesell, mein liebster Hort, ein neujahrslied aus ca. I 300," Arcbiv für Musikwissenschaft IV (1922), 4I3-I9. II. "Die Mondseer Liederhandschrift und Herman, der Münch von Salzburg," $\mathrm{V}$ (I923), I I-30. See I4-10.

36. F. Vogt, Des Minnesangs Frübling, Leipzig, Hirzel, i 888. (30 Aufl. I950).

37. Johann Christof Wagenseil, Bucb von der Meistersinger boldseligen Kunst... Pp. 433-575 of his De Sacri Rom. Imperii libera Civitate Noribergensi Commentatio. Nuremberg, I697.

38. Wehrli: Max Wehrli, Deutsche Lyrik des Mittelalters. Zürich, Manesse Verlag, I 955 .

39. zfdA: Zeitschrift für deutsches Altertum und deutsche Literatur.

40. zfmw: Zeitschrift für Musikwissenschaft.

4I. Zitzmann: Rudolf Zitzmann, Die Melodien der Kolmarer Liederhandscbrift. Würzburg, Triltsch, I944.

Further bibliography, dealing with relations of Minnesinger, Trouveres, and Troubadours, listed under 6r. 
I. DER WILDE ALEXANDER

(Meister Alexander)

XIII C

Rudolf Haller, Der wilde Alexander. Würzburg, Triltsch, I935 Gunther Hase, Der Minneleich Meister Alexanders. Halle, I92I. DTO XLI

I. Ach owê, daz nâch liebe ergât Jena 25b; Wien 270r, f. 49 HM IV, 78 \% ; JHSB I, $46 \%$, II, I $4 d$, I I $3 d$; DTO XLI, 86

CE: KLD I, I 3

2. Ein wunder in der werlde vert Jena $22 a$ HM IV, 783 १; JHSB I, 4I १, II, I 2 d CE: KLD I, 2

- Ein trurechiches...: I-4

3. Hie vor dô wir kinder wâren Jena $24 \mathrm{~d}$ HM IV, $784 \uparrow$; JHSB I, 46 १, II, I 3 d, II $8 d$; GTM III, $8 d$; GMMA $220 d$; Kuhn-Reichert I 53 d HM III, 30; Wehrli, 435; CE: KLD I, I 2

4. Min trûreclîchez klagen

Wien: „Das ist des wildyn Alexandyrs leych"

Jena 25d; Wien 270I, f. 44

HM IV, 785-90 १; JHSB I, 47 \%,

II, I4 d ; DTO XLI, 83-86 d;

Kuhn-Reichert I 54 d

HM II, 364-65; CE: KLD I, Is

Stanza I $\mathrm{r}$ : Nu memet war, diz ist der schilt

MGDM I, I73

Leich

5. Siôn trûre

Jena $24 b$

HM IV, 784 १; JHSB I, 45 १, II, I 3 d;

MGDM I, I67

HM III, 3O; CE: KLD I, IO 
TEBM 29; BMKH I8I

I. Heiliger geist, din kraft mit aller guete

„In meister Anckers tone”

Colmar 779

Runge, Colmar, 167

BMKH 548

3. MEISTER BOPPE

XIII $C$

тЕмв 30-3 I; вмкн 166. Meister Boppe and Der Starke Boppe (xIV c) often confused.

G. Tolle, Der Spruchdichter Boppe, Versuch einer kritischen Ausgabe seiner Dichtungen. Progr. Sondershausen, 1894

I. Ich weiss nit, ob der hymmel hanget oder swebt

„Gesang in dez starcken

Boppen hofedone"

Colmar 555; APsB 60

Runge, Colmar, I 36 ; JHSB II, r97 d

H. Enke, „Der 'hofedon' des Meister

Boppe", Festschrift für Max Scbneider.

Leipzig, I955.

2. Ohoer unde starker almechtiger Got

Jena I I Ic

HM IV, 83 I ๆ; JHSB I, I92 †, II, 69 d;

Runge, Colmar, I $37 \uparrow$

PMDL I, 217

- BURK MANGOLT -

Composer of music to poems of Hugo von Montfort, q.v.

- EHRENBOTE VOM RHEIN : SEe REINMAR VON ZWETER -

- FRAUENLOB: See HEINRICH VON MEISSEN -

4. MEISTER FRIEDRICH VON SUNNENBURG

$2 / 2$ XIII C

I. Ein richer kunik hiez Kosdras

Jena $70 b$

HM IV, $807 \uparrow ;$ JHSB I, II 9 , II, 40 d 
2. Ich wil singen

Jena $72 \mathrm{~d}$

HM IV, $808 \uparrow$; JHSB I, I 23 १, II, 42 d

HM III, 78

3. Nu merke ho und edele man Jena $7 \mathrm{Ib}$ HM IV, 808 १; JHSB I, I 2 I १, II, 4I •

4. O wol dir, Welt, o wol dir hiute und je mermere wol Jena $63 \mathrm{c}$ HM IV, 806 ๆ; JHSB I, IO8 १, II, $39 \uparrow$

See also 20-2

5. MEISTER GERVELIN

I. Drivaltik name der Goteheit Jena $3 \mathrm{Ib}$

HM IV, 792 १; JHSB I, 58 १, II, 2I d

HM III, 35

2. Golt von Arabie ist guot, daz darf ouch nieman strafen Jena $35 \mathrm{~b}$

HM IV, 792 १; JHSB I, 6I १, II, 22 d

HM III, 37

$$
\begin{aligned}
& \text { 6. DER GUTER } \\
& \text { XIII C }
\end{aligned}
$$

I. Hie vor ein werder ritter lak Jena $38 \mathrm{~b}-39 \mathrm{a}$ HM IV, $794 \uparrow$; JHSB I, 67 १, II, 24 d; HM III, 4I

BMKH I 82

$$
\text { 7. HARDER, KONRAD }
$$$$
\text { XIV-XV C }
$$

I. Ayn schone meit dorchsonet „In des Harders korwyse, der guldin schilling, ist ein leych" Colmar 33

Runge, Colmar, $20 \uparrow$ BMKH 192 
2. Man höret aber richen schal

"Des Harders guldin rey"

Colmar 36

Runge, Colmar, 201

BMKH 197

3. Mary, kungynn ob allen kunne

"In dem Harder sin hoffwyse"

"Colmar, 845; APSB 90, „süsse ton”

Runge, Colmar, 1839

8. HEINRICH VON MEISSEN (FRAUENLOB)

$$
\text { I } 260-\text { I } 3 \text { I } 8
$$

TEBM 36; DTO XLI; BMKH I68-75

L. Ettmüller, Heinrichs von Meissen des Frauenlobes Leiche, Sprüche, Streitgedicbte und Lieder. Quetlinburg und Leipzig, i 843. Helmuth Thomas, Untersucbungen zur Überlieferung der Spruchdichtung

Frauenlobs. (Palaestra 217). Leipzig, 1939. Mss., pp. 2-146.

A. E. Kroeger, The Lay of Our Lady. Translated from the German of Frauenlob. St. Louis, I 877. Ludwig Pfannmüller, Frauenlobs Marienleich.

Strassburg, Trübner, I9I3. Margarete Lang, Der Minnesinger Frauenlob. Mainz, I95 I. Kirsch, Frauenlobs Kreuqleich. Diss. Dillingen, I930. H. Enke, „Der vergessene Ton Frauenlobs”, Musikforscbung IV (I95 I). K. H. Bertau, R. Stephan, „Wenig beachtete Frauenlobfragmente," zfdA Lxxxvi (1955-56), 302-20.

\begin{tabular}{|c|c|c|}
\hline & & \\
\hline lwyse 2 I & Hagenblu 49 & Spiegelwyse 30,50 \\
\hline Blauen 3 & Heylygin crucys leich 55 & Spruch I $9,20,39,40$, \\
\hline Bluenden 23 & Hunds Fuss 57 & 43 \\
\hline Frauenleich 7 & Huntwyse 36 & Suessen ro \\
\hline Froschwyse 9 & Jar Weiss is & Tagweiss 34 \\
\hline Dan I 8 & Kupfer 32 & Thon thon 58 \\
\hline Gecronten I $3,41,44,56$ & Langen 25,29 & Überzarten $\mathrm{I} 4$ \\
\hline Geilen 4 & Leit I 2 & Vergessnen 2,48 \\
\hline Geschwinden 24 & Mynnekliche leych 42 & Verholn 5 \\
\hline hen $1,16,38,53$ & Newen 33, 52 & Vluk 46 \\
\hline Gruntwys I I & Ritter Weiss 22, & Würgindrossil $6,8,54$ \\
\hline Gulden 3 I & Slosshort 27 & Zarten $17,28,45$ \\
\hline Gulden Radweise 35, 5 I & Spätten 26 & Zugweiss 47 \\
\hline
\end{tabular}

I. Alle so hie betrüglich "In gruenen thon Frauenlobs" Berlin 24, f. 235 , f. 483 (words only) HM IV, $927 \uparrow$ 
2. Als Paulus die Jünger Christ versehret

„Im vergessnen thon Frauenlobs"

Berlin 24, f. 244

HM IV, 930 १

3. Auff ein Zeit gut Nachpauren sich „Im Blawen thon Frauenlobs”

Berlin 24, f. 24IV; APSB I7

4. Dem Son Gottes almechtig „Im Gailen thon Frauenlobs" Berlin 24, f. 242v, 494 (words only); APSB 20

5. Der kungynn ich ob allen kungyn dienen will

„Im verholn don Frauenlobs"

Donaueschingen 257; Colmar I55;

Berlin 24, f. 249r (no words)

Runge, Colmar 769

6. Dez hymelz ercztenye

„Dis ist Vrouwinlobis in dem

wurgindrossil dy dry"

Wien 270I, f. I7; APSB 5

DTO XLI, 68

Do kuninc Alexander: see 8-29

7. Ei ich sach in dem trone

Colmar 19; München, Fragment M;

Königsberg fragment; Wrocław I. Q. 368

„Diss ist unser frawen leich oder der guldin flügel zu latin Cantica Canticorum. Stent ie zwey lied in eym ton und in eym gemesse und sint der töne XXII der lieder XLIIII"

Runge, Colmar 3-16 \%, facsimile I; NOHM II, $258 d$; zfdA LXXXVI, 307-I I Record: History of Music in Sound, ii, side I I, Marienleich 
- Ein snider sneit mir min gewant „Aus unser Frouwen leich' Frauenlobs"

Wien 270 I, f. 2 DTO XLI, 57 d

8. Ein burger sass zu bretten „Im Würgendrüssel Frauenlobs” Berlin 24, f. 228v HM IV, 9291

9. Eyn ey gelich, ist synevel "In der froschwyse Frawenlobs" Donauschingen 278; Colmar 136 ; Berlin 24, f. $238 \mathrm{v}, 487$ (no music); APSB I5 Runge, Colmar $73 \uparrow$ BMKH 29I

- Ein sneider sneit mir...: see 8-7

Io. Ein wort, daz ist ein wesen "In Frawenlobes suessen ton" Colmar I 86; Berlin 24, f. 25 I v Runge, Colmar $88 \uparrow$

I I. Ein wort daz wont ym allerhochsten trone "In Frauwenlobs gruntwys" Colmar 194; Berlin 24, f. 246r, 497 (words only); APSB 23 Runge, Colmar 909

- Ewiger, starcker, hoher, almechtiger Got: see 35-6

I 2. Fruchtenbernde frucht der welte trost

„In Frauwenlobs leyt ton”

Donaueschingen 3 I 5 ; Colmar I 88;

Berlin 24, f. 229v; APSB 8 .

Runge, Colmar $89 \uparrow$

I3. Gar starc bekant ist der helfant

„Im gecronten ton Frauenlops"

Donaueschingen 280; Colmar I 57 ;

Berlin 24, f. 224v

Runge, Colmar $78 \uparrow$ 
I4. Heyliger geist, herlucht min synne kamer

"In Frauwenlobes überzarten don"

Donaueschingen 236; Colmar 28;

APSB I

Runge, Colmar i 6

BMKH I 88

I s. Herr... beschriben hat

"In der Jar weiss Frauenlobs"

Berlin 24, 238r; APSB I4

I6. Her Symeon der wyse

"In Frauwenlobes gruen don"

Donaueschingen 3 I 2 ; Colmar 2 I 2 ;

APSB I 2

Runge, Colmar Io2

I7. Ich hoer' des vater lere jehen

„Im zarten don"

Jena I Ioc; Berlin 24, f. 232 r (no

words), 242 ; APSB 6

HM IV, $830 \uparrow, 927$; JHSB I, I90 १,

II, 68

HM III, I 52

I8. Ich kam geritten uff ein felt

"In Frauwenlobs dan ton"

Donauschingen 275; Colmar I 28

Runge, Colmar 7I

19. Ich mane dich, Gotes sun Jhesus, der tyfen wunden rot

Wien 270 I, f. 20

DTO XLI, 69

I of s Sprüche

20. Ich mane dich grozer note vumphe, muter, reyne mayt

Wien 2701 , f. I9

DTO XLI, 69 d

I of 5 Sprüche 
2I. Ich sach in einen garten gan "In der anckelwyse Frauwenlobs" Colmar 24I; Berlin 252r (no words) Runge, Colmar Ios 1 ; $\mathrm{zfdA}$ LXxx (1943), $9^{8}$ d ; GTM III, I4 d

- Im andern Buch Samuelis: 8-24

22. Im geschicht schreiber Justino "In der Ritter Weiss Frauenlobs" Berlin 24, f. $243 \mathrm{r}$

23. Im Mayen frü ein Jungling kun „Im Blüenden thon Frauenlobs" Berlin 24, f. 240v; APSB I9 d

24. In der Fasten kam zu ein dorff Pfaffen „Im geschwinden thon Frauenlobs" Berlin 24, f. 236v; APSB I 3 d

29. In der offenbarung Johannes haben wir „Im langen thon Frauenlobs" Berlin 24, f. $225 \mathrm{v}$ HM IV, 926 ?

26. In einer weitbekanten stat „Im spätten thon Frauenlobs" Berlin 24, f. 245r, 496 (words only); APSB 2 I

27. In Gottes schoss gesehen wart in hoher art „Dyss ist Frauenlobs taugenhort oder sin slosshort"

Colmar 43

Runge, Colmar 28-49 ?

BMKH 204

Frauenlob or Peter von Reichenbach? See BMKн I69, I8I on autborship 
28. Jacob der fuenfft ein kuenig in Schotlande

„In der Zugweiss Frauenlobs”

Berlin 24, f. 239v; APSB I 6

HM IV, 928 ?

29. Jesayas der schrybet so, der Gottes

knecht

„Im langen ton Frauwenlobes”

Donaueschingen 249; Colmar 94;

APSB 4

Runge, Colmar $6_{7}$

2nd stanza: Do kuninc Alexander myt

vulkomner macht, JHSB II, I95

30. Johannes der sach also fron

„In Frauwenlobes spiegelwyse"

Colmar $\mathrm{I}_{3}$, I86; Berlin 24, f. 246v,

498 (words only); APSB 24

Runge, Colmar $82 \uparrow, 88 \uparrow$

$3 \mathrm{I}$. Jung son und alter vater

"In Frauwenlobs guldin tone"

Donaueschingen 255; Colmar I 39;

Berlin 24, f. 230; APSB 2 d

Runge, Colmar $74 \uparrow$

32. Karheit halbet so hatte

„Im Kupfer thon Frauenlobs”

Berlin 24, f. 234V, 48 I (words only);

APSB I I

33. Klar thut Vicencius berichten

„Im newen thon Frauenlobs"

Berlin 24, f. 227r; APSB 7

HM IV, 929 ?

34. Künig Wunibald in Burgund

"In der Tagweiss Frauenlobs"

Berlin 24, f. 233r; APSB 9

35. Lucas spricht klar

"Gulden Radweise"

APSB Io 
36. Maria kunigynne „In der huntwyse Fr." Donaueschingen 283; Colmar I6I Runge, Colmar 80, 9 ; JHSB II, 196

37. Maria, muter, reine meit „In Frauwenlobs Rytter wyse" Donaueschingen 313; Colmar 222; Berlin 24, f. 243r; APSB I 8 Runge, Colmar I03 1

38. Myn vroud ist gar czugangyn „In der grunen wyse"

Wien 2701, f. I7 DTO XLI, 67 d ; SGMB I 4 d Spruch

39. Nu geseygyn mich hut Got vater, sun und ouch heliger geyst

Wien 2701 , f. 19 DTO XLI, 68 Spruch

40. $\mathrm{Nu}$ wil ich nymmermer vor zcwiln an dem hemilrich

Wien 270 I, f. 2 I

DTO XLI, 70

Spruch

4I. Nun aber war ein Brunnen daselbst an dem Ort

„Der meisterliche Hort, in vier gekroenten Toenen... das ander Gesetz, im langen Ton Heinrichs Frauenlobs" (Other 3: 9-2, 25-9, 35-10) APSB 3

HM IV, 9339 ; Wagenseil 5549

42. O wip, du hoer eren haft "Das ist der mynnekliche leych" Wien $270 \mathrm{I}, \mathrm{f} .34$ DTO XLI, $77-83$ d 
43. Richer man, Got lech dir gut, her hot dirs nicht gegebn

Wien 270 I, f. 22

DTO XLI, 70

Spruch

44. Sint frolich, fraw, mit zuchten gein dem meyen

„Her Frauwenlobs gecronter rey”

„In dem gecronten Rey weiss

Frauenlobs"

Colmar 4I ; Donaueschingen 245;

Berlin 24, f. 2 sor (no words)

Runge, Colmar 259

BMKH 201

45. Sun, du bist sun, sun, ussertracht

„Im zarten ton Frauwenlobs”

Donaueschingen 287; Colmar 196

Runge, Colmar 91 9

Wehrli 468

46. Swa sich diu tugent erbiutet

„Im vluk don"

Jena ıo6c

HM IV, $828 \uparrow ;$ JHSB I, I $84 \uparrow$, II, 67 。

HM III, I 4I

47. Us erentricher porten wart gesendet

"In Frauenlobes zuegewyse"

Colmar 236

Runge, Colmar Io4?

48. Usz alter ee schribet man uns besunder

„Im vergessen ton Frauwenlobs in antiquo dictamine"

Colmar I66; Berlin 24, f. 244, 495

(words only); АPSB 22

Runge, Colmar 82

49. Von ainer edlen Frawen

„In der Hagenblu weiss Frauenlobs”

Berlin 24, f. 247r; APSB 25 
50. Weil die Christen verfolget hart „Im Spiegelton Frauenlobs”

Berlin 24, f. 246

HM IV, 93I $\uparrow$

5I. Wer der trau Gott

"In der gulden Radweiss Frauenlobs”

Berlin 24, f. 230v; APSB IO d

52. Wer hallff Adam uss not in fure "In dem nuwen ton Frauenlobs" Donaueschingen 252; Colmar 176 Runge, Colmar 831

53. Wer kante Gotes krefte „Im gruenen don” Jena ro8a; Wien 270r, f. I7a HM IV, 829 १; JHSB I, I 86 १, II, 68 d; DTO XLI, 87 HM III, I44

54. Wir leben hie in sunden „Im wurgendruessel Frauwenlobs" Colmar i 6 Runge, Colmar $70 \uparrow$, xvIr

55. Wo wundir werndir suz ursprink „Das ist des heylygyn cruecys leych" Wien 270I, f. 22-23 DTO XLI, 7I-77 d Frauenlob or Regenbogen? See 35-4

56. .

"In der Hunds Fuss weiss Frauenlobs" Berlin 24, f. 248r (no words)

$57 \ldots \ldots \ldots$

"Im Thon thon Frauenlobs"

Berlin 24,f. 25 Ir (no words)

See also 36-6, 4I-8

I 2 
TEBM 37; BMKH I80

9. HEINRICH VON MÜGELN

$$
\text { I320?-I } 370 \text { ? }
$$

Karl J. Schöer, Die Dichtungen Heinrichs von Mügeln, nach den Hss. besprochen. (Sitzungsberichte der Akademie der Wissenschaft zu

Wien. Phil.-hist. Klasse, LV (I 867), 45 Iff.). Heinrichs von Mügeln kleineren Dichtungen (Deutsche Texte des Mittelalters), 1959.

I. Einen gecronten reyen

„In meynster Heinrich von Mugelin

traumton

Colmar 632; APSB 27

Runge, Colmar 140

2. Genesis am neun und zwanzigstens uns bericht

„Der meisterlich Hort, in vier gekroenten Toenen. Das erste Gesetz im langen Ton Heinrich Muglins" (Other 3 : 8-4I, 25-9, 35-10)

HM IV, $932 \uparrow$; Wagenseil 554

See NOHM II, 259

3. Hillf Herr Gott der Heyligen dein „Hoff ton”

APSB 29d

4. Wer nu der bybelbuch

„Im kurtzenn Mueglins”

Colmar 6II; APSB 30

Runge, Colmar I $39 ?$

5. Wer tichtet, und gesach nie warer kunste grunt

„Hie volget meinster Heinrich von

Mügelins gedichte, zuerst in sim

langen tone."

„Heinrich Müglings langen ton.

Ist der 4 gekroenten einer"

Colmar 592; Wien 2856; APSB 26

Runge, Colmar I 37 , I 38 १

See 9-2 


\section{Wilt du mentschart}

„In meinster Heinrich von Müglins

grun ton"

Colmar 640; Wien 28 56, f. 247; APSB

28

Runge, Colmar 1401

See 28

IO. HEINRICH VON OFTERDINGEN

(Before I 250)

BMKH I5 8; TEBM 37: "a mythical figure"

A. Strack, Zur Geschicbte der Gedichte vom Wartburgkriege. Diss. Berlin, I 883

I. Das erste singen daz hie tut

(Daz erste singen nu hie tuot)

„In dem gekauften oder in den fursten

ton Heinrichs von Offertingen

von erst die zwene kriege"

„Kreig von Wartburg”

"In des Düringe herren ton"

„In des edelen vursten dhon

von Duryngelant..."

Colmar 756; Jena I $23 \mathrm{~d}$

HM IV, 8437 ; Runge, Colmar, 1659 ;

JHSB I, 2 I 4 १, II, 83 d, I99 d

HM II, I, 3

- HEINRICH DER TUGENDHAFTE SCHREIBER : 43 -

- DER HELLEVIUR: see HÖLLEFEUER -

II. DER HENNEBERGER

I. Swer da gerne ritter wirt mit hoher wirdikeit

Jena $36 c$

HM IV, 793 १; JHSB I, 63 १, II, 23 d

HM III, 39 


\section{I2. HERMANN DER DAMEN}

Helene Onnes, De gedichten van Hermann der Damen. Proefschr., Groningen, I9I3

I. Der aller wunder meister ist Jena i i 8 a HM IV, $839 \uparrow$; JHSB I, $205 \uparrow$, II, 79 d HM III, I 62

2. Ein lop sing' ich dir ze prise Jena $\mathrm{i} 2 \mathrm{xb}$ HM IV, 84I १; JHSB I, 2 IO १, II, 8 I d HM III, 167

3. Het' ich al der werlde hulde Jena II $\mathrm{d}$ HM IV, 839 १; JHSB I, 204 १, II, 79 d; GTM III, I 3 d ; Lang-Salmen $4{ }^{\circ} d$ HM III, $\mathrm{IG}_{2}$

4. Ich male uf des sanges sims Jena I $22 \mathrm{C}$ HM IV, 84I १; JHSB I, 2 I 2 १, II, 82 d HM III, 169

5. In dirre wise ich singen wil Jena $119 \mathrm{~b}$ HM IV, $840 \uparrow ;$ JHSB I, $207 \uparrow, 80$ 」 HM III, I64

\section{Ir Kristenen, alle schriet} Jena I I 3 d-I I 7 d HM IV, 832-39 १; JHSB I, I 98 १, II, 7 I • HM III, I60

I3. HERMANN DER MÖNCH VON SALZBURG XIV C

BMKH I 84; Wehrli: "eigentlich zwei Hofdichtern” F. A. Mayer und H. Rietsch, Die Mondsee-Wiener Liederbandscbrift und der Mönch von Salzburg, Acta Germanica III, 4-Iv. Berlin, 1894-96. 


\section{Ave, Balsams creatur}

„Des Munchs von Salczburg guldin abc"

Colmar 653; München, Cgm. 715, f. 46; Wien 2856, f. 166

Runge, Colmar, I45-I 50 ?

HM III, 468

2. Beschnytten wirdiclichen

"Des Munches Cisiojanus die jarwyse" Colmar 662

Runge, Colmar, I 54 १; GFML I94 d

3. Die nacht wirt schier des hymmels gast

"Dyss ist des Munchs korwyse"

Colmar 658; Berlin 25, p. 31 ; APSB 87

Runge, Colmar, I 539

4. Gar lys, in senfter wys

„Dyss ist daz taghorn dez Munchs von Salzpurg"

Colmar 657; Wien 2856, f. 186

Runge, Colmar, I 50 ; G. Adler,

Vierteljabrschrift für Musikwissenschaft

II (1886), 310-I 2 १, d; GMMA 225

5. Her Got almechtig, dry person

„Darnach manche von Salzburg des Munchs zarter don"

Colmar 644; München, Cgm. 715 ,

f. 7 I 5 ; Wien 2856, f. 178 ; Berlin 24,

f. I 58 (no words)

Runge, Colmar, I4I $_{1}$

6. Ich bit dich, geber guter dinge „In des Muenchs suessen ton. etlich sprechen korwyse"

Colmar 652

Runge, Colmar, I44 9 
7. Kum senfter trost, heiliger geist "Im Munch von Salczburg langen tone" Colmar 646; Donaueschingen 222 ; Wien 2856, f. 182; München, Cgm. 715, f. 162 ; APSB 88

Runge, Colmar, 1429

8. Magt hochgeborn von dem geslecht Jesse "In des Munches hoffdone" Colmar 645; München, Cgm. 715 , ff. 175, I77; Wien 2856, f. 242; Berlin 24, f. I6o (no words) Runge, Colmar, 1429

9. Maria, wyss gegrüsset! „In des Munchs von Salczburgk kurzen ton" Colmar 66I ; Berlin 24, f. I6I (no words) Runge, Colmar, 1539

I0. Mein traut gesell, mein liebster hort MGDM I, I 80 d O. Ursprung, AfMW IV (1922), 4I3 १, d

II. Mues ich mich von dir schaiden AfMW V (1923), 29d

I 2. Myn liebste fraw, in lieber acht "Das nachthorn"

Colmar 658; Wien 2856, ff. 185, 245 Runge, Colmar, Is 2 \%; Adler, op. cit. 4, supra, 3I 2-3 Is 1 ,

13. Wol mich wart! ain frëwelein czart AfMW V (1923), 29d

$$
\begin{aligned}
& \text { I4. DER HÖLLEFEUER } \\
& 2 / 2 \text { XIII C }
\end{aligned}
$$

I. In diser wise daz erste liet Jena zoa HM IV, 79I १; JHSB I, 56 १, II, 20 d HM III, 33 


\section{I5. HUGO (VIII, GRAF) VON MONTFORT \\ I $375-I 423$}

Paul Runge, Die Lieder des Hugo von Montfort mit den Melodien des Burk Mangolt. Leipzig, Breitkopf und Härtel, 1906. Pp. 75. (Texts, original notation, modern transcription, I facsimile).

J. E. Wackernell, Hugo von Montfort. I88 I.

Karl Bartsch, Hugo von Montfort (Bibl. Litt. Verein Stuttgart, CXLIII), Tübingen, 1879 .

E. Jammers, „Die Melodien Hugos von Montfort”, AfMw xIII (1956), 217-35.

Ms: Heidelberg, Univ. Bibl., Cod. Pal. germ. 329 (folios given below).

\section{„Die weysen hat gemachen \\ Bürk Mangolt \\ Unser getrewer knecht." \\ Hugo von Montfort}

I. Des hiemels vogt und hochster keiser

f. 50

Runge, 65 1, 68

Bartsch: „Unecht”, but see Runge 4

2. Fraw wilt du wissen was es ist

f. $2 \mathrm{IV}$

Runge, 42 १, 43 d ; Wolf, Handbuch

derNotationskunde, I, 177-78 d, facsimile

3. Fro welt ir sint gar hüpsch und schön

f. $35 \mathrm{r}$

Runge, $45 \uparrow, 4^{6} d$, facsimile, frontispiece

MGDM I, I 90 d

4. Ich fragt ein wachter ob es wer tag

f. IIr

Runge, 26 1, 27d

Wehrli, 332

5. Ich fröw mich gen des absentz kunst

f. Iov

Runge, $23 \uparrow, 23 \mathrm{~d}$

6. Ich var uff wag des bittern mer

f. $\mathrm{I} 3 \mathrm{r}$

Runge, $37 \uparrow, 38$ d 
7. Könd ich ein gedicht volbringen

f. $48 \mathrm{v}$

Runge, $579,59 d$

Bartsch: „Unecht”, but see Runge 4

8. Mich straft ein wachter des morgens

frü

f. IIV

Runge, 29 १, 31

9. Sag an wachter wie was es tag

f. I $2 \mathrm{~V}$

Runge, 341,35 .

Io. Weka wekh die zarten lieben

f. $46 \mathrm{r}$

Runge, $20 d, 52 \uparrow, 54 d$

I6. DER KANZLER

Fl. I 300

TEBM 32; BMKH 167

H. Krieger, Der Kanqler. Ein mbd. Spruch-und Liederdicbter um 1300.

Diss. Bonn, I93 I

I. Am zwei und viertzigisten

beschreibt Esaias fein

„Im gulden Ton Cantzlers"

Berlin 25, p. I 4

HM IV, 925

2. David, din blick sint worden war "Item in Kanczlers langen tone"

Colmar 592

Runge, Colmar, I 349

3. Frou Minne, getiuret si din nam

"In des Kanczlers sussen done”

Colmar 554

Runge, Colmar, I 359

HM III, 454; KLD I, 2 I 
4. Got schepher aller dingen

Stanza I 2: Was wird und hôher êre

"In des Kanczlers hohen gulden tone"

Colmar 545; APSB 62

Runge, Colmar, 1309

KLD I, I 87; BMKH 486

Stanza I 5 : Jôhannes in dem trône

sach Got in sîner majestât

„In dysem hohen guldin Canczler...”

KLD I, I 87; BMKH 488

5. Ich weiss ein keyserliche meyt "In des Kanczlers hofdone"

Colmar 553; Berlin 24, f. I57v

(no words)

Runge, Colmar, I35 \ HM IV, 93 I

BMKH 55

- Johannes in dem trône...: 16-4

- Was wird und hoher ere: $16-4$

I7. MEISTER KELIN

2/2 XIII C

I. Ein künik in sime troume sach Jena $16 \mathrm{c}$

HM IV, 780 १; JHSB I, 3 I $\uparrow$, II, 8

HM III, 20

2. Ez ist vil maniger here

Jena I 8b; Basel, N.J. 3, nr. I45

HM IV, 78I १; JHSB I, $34 \uparrow$, II, IO

HM III, 22; JHSB II, I 54, re Basel frag-

ment

3. Vil riche saelde, mich nimt immer wunder

Jena I $7 \mathrm{c}$; Basel N.J. 3, nr. I45

HM IV, 78I १; JHSB I, 33 १, II, 9 d

HM III, 2 I 
I8. KLINGSOR

XIII C

BMKH I 8 ; TEBM 39:, ,a mythical figure”

I. Ein edelbaum gewachsen ist

„In Clingesores swarczen ton"

Colmar 680; Jena I 34c (text only);

APsB 59 („Klinges Uhr”)

Runge, Colmar, I 597 ; JHSB II, I98

HM III, I8 I, „Kriek von Wartberk”

19. KONRAD VON QUEINFURT

$$
- \text { I } 382
$$

I. Du lenze gut, des jares tiurste quarte

Leipzig, Univ. Bibl., ms. I 305 , ff. 107-

I IO

MGDM I, I 77

20. KONRAD VON WÜRZBURG

BMKH I 64

$$
\text { I } 225 \text { ? }-1287
$$

Konrad von Würzburg, Partonopier und Meliur... Lieder und Sprüche, hrsg. von Karl Bartsch. Wien, i87 I

E. Schröder, Konrads von Würzburg kleinere Dichtungen, III. Berlin, Weidmann, 1959 .

I. Auss der dieffe schrei ich zu dir „Im abgespitzten Ton Conr. von

Wirtzburg",

Berlin 25, p. 23 ; APSB 64

HM IV, 924 ?

2. Ave, ich lobe dich, reine meit

„In Cunrads von Wirczburg nacht-

wyse. Alii dicunt esse In Frider. von

Suneburg sussem don"

Colmar 526

Runge, Colmar, I 27

3. Ave Maria, kusche maget stete

"In Cunradz von Wirczburg morgen-

wyse"

Colmar 5 I 2

Runge, Colmar, I 269 
4. Der nit sin vaz vil tunkel verwet, als ein bleich gehilwe Jena Iorb HM IV, $827 \uparrow$; JHSB I, I 68 १, II, 66 。

5. Dez soltu clein geniessen „In Conratz von Wirtzburg kurczen oder im werden don"

Colmar 528

Runge, Colmar, I 28 ; GTM III, 9 BMKH 465

6. Er mag vil lichte witze han „In meinster Cunratz von Wirczeburg blawen ton"

Colmar 54I; APSB 45, ascribed to „Bartel Regenbogen, des Schmides” Runge, Colmar, I 29

BMKH 484

7. Hochvart ist worden also gross „In meinster Cunrades von Wirczburg auspis"

Colmar 506

Runge, Colmar, i 269

BMKH 456

8. Waz in dem paradys ie wart gebildet und gemachet

"In Cunrads von Wirczburg hoff don"

Colmar 53I; APSB 63

Runge, Colmar, I 28

BMKH 467

9. Woluff ir geist, hin uber mer

"Meinster Cunrads guldin reyel"

Colmar 43

Runge, Colmar, $27 \uparrow$

- KRIEG VON WARBURG: see IO-I; I8-I; 56-I - 
BMKH I 84; TEBM 39

L. Köster, Albrecht Lesch (Ein Münchener Meistersänger d. 16 Jbs.)

Diss. München, 1937

I. Ave Maria, dich lobet musica „Diss ist Leschen guldyner reye” Donaueschingen 219; Colmar 37 Runge, Colmar, 2I 9

2. Daz recht ist manigfeltig krump "Dyss ist in Leschen hoffwyse" Colmar 837 Runge, Colmar, $179 \uparrow$

3. Gott herr, din ewikeite "Leschen getichte. Sin cirkelwyse"

Colmar 831; APSB $73 d$; Berlin 25, p. 38 (no words) Runge, Colmar, $177 \uparrow$

4. Ich lob die reinen frauwen zart "In Leschen fur wyse" Colmar 835; Berlin 25, p. 36 (no words); APSB 72 („Feuerw.”) Runge, Colmar, 1781 BMKH. 84

5. Wer nympt mit lieb das hochste gut "Leschen sang wyse" Colmar 839; APSB 7 I Runge, Colmar, 1809

6. Zuch durch die wolken, myn gesang "Leschen tagwyse" Colmar 843 Runge, Colmar, i 8 I BMKH 87

I. O Maria, du reine meit "Ein anders sinem senften ton" Colmar 792 Runge, Colmar, $170 \uparrow$ BMKH 79 
2. Was sol ein meder uff daz mat

"In des Lieben jarwyse"

Colmar 790; APSB 9I

Runge, Colmar, I69

3. ..... blurred

„In der Radweis Lieben von Gengen"

Berlin 25, p. 40

23. DER LITSCHAUER

I. Man sach hie voren die alten herren eren pflegen

Jena $42 a$

HM IV, $796 \uparrow$; JHSB I, 73 १, II, 26

HM III, 46

BMKH I 86

24. MAGISTER HUIUS LIBRI (SC. COLMAR)

I. Ich singe gerne lyse

„Im unerkanten ton. V Liedt..."

Colmar 492; Donaueschingen 21 2;

APSB 93, ascribed to „Wolf Nestler

von Ulm"

Runge, Colmar, 23

25. DER MARNER

Fl. I 230-I 244

TEBM 40; BMKH I60

Philip Strauch, Der Marner. Strassburg, Trübner, I876 (Quellen und Forschungen, xIV)

I. Das auf Erd nicht schedlicher sag „Im Kreutz thon Marners”

Berlin 24, f. $256 r$; APSB 32 d

2. Esaias der Prophet spricht

„Im suessen thon Marners"

Berlin 24, f. 259 r (no words); APSB

$34 d$ 
3. Ich lob ein meit ubr alle lant „Der propheten dantz ein eygen geticht des Marners"

Colmar 489; Berlin 24, f. 25 2v;

APSB 36

Runge, Colmar I 2 I 9

BMKH 47-49; Strauch 63

4. Ich mercke, daz diu sonne

"In Marners kurczem oder hofedone"

Colmar 494; Berlin 24, f. 257r; APSB 33

Runge, Colmar I 25 ?

вмкн 444; Strauch I 30 , as „Anhang”

to Marner's $\mathrm{Ob}$ allen frouwen frouwe;

Strauch 60-6I

5. Im heiligen Matheo klar

"Im langen thon Marners"

Berlin 24, f. 254V

HM IV, 9239

See $25-8$

6. In ein fuerneme stat hinkam

"Im gulden thon Marners"

Berlin 24, f. $258 \mathrm{r}$; APSB 35 .

HM IV, 9231

Strauch 59

7. Ir schauwent an die clein ameiss

"Dyss par stet in Marners tone"

Colmar 490

Runge, Colmar $122 \uparrow$

BMKH 442

8. Maria, muter, reine meit

"Dyss ist des Marners lange ton"

„Im langen Marner ton. Ist der

4 gekroenten thone einer"

Colmar 447; APSB 3 I

Runge, Colmar i i9 १, I 20 d

Strauch 61-63

See 25-5, 25-9 
9. Nach den aber das werk verricht „Der meisterliche Hort, in vier gekroenten tonen... Das dritte Gesetz, im langen ton Ludwig Marners" (Other 3:8-4I, 9-2, 35-10) HM IV, 934 ; Wagenseil 554 ? See 25-5, 25-8

BMKH I8I

26. MEFFRID

XIV C

I. Hercz unde synn, nu muewe dich „Meinster Meffryds geticht" Colmar 794; Engelberg Runge, Colmar, I7I $\uparrow$; Schweizer Jabrbuch f.M.W., 1928, p. 64f.; Kirchenmusik Jabrbuch XxI (1908), 49 BMKH 550

\section{MEISSNER \\ $2 / 2$ XIII C}

A. Frisch, Untersucbungen über die verschiedenen mbd. Dichter, welche nach der Ueberlieferung den Namen Meissner fübren. Diss. Jena, I 887

I. Almehtik Got, barmunge rich, sich hie nider in diz elende Jena 94 a HM IV, $824 \uparrow$; JHSB I, I $57 \uparrow$, II, 62 d HM III, IO2

2. Daz sank daz hoeste si in himele unde uf erden Jena 9id HM IV, 823 १; JHSB I, I 53 १, II, 60 . HM III, 99

3. Got, aller saelden ane vank, dir si genigen Jena 8 ra HM IV, 8I7 १; JHSB I, I $35 \uparrow$, II, 53 d HM III, 86 
4. Got, der ging durch uns einer jemerlichen gang

"Der Michsener in sim gedicht"

Colmar 797

Runge, Colmar, I 719

5. Got der hat uns nach im gebildet Jena 94d

HM IV, $825 \uparrow ;$ JHSB I, I 58 , II, 62 。

HM III, IO3

6. Got ist gewaltik

Jena $87 \mathrm{~b}$

HM IV, $820 \uparrow$; JHSB I, I46 १, II, 56 d ;

Riemann 269

HM III, 94

7. ...hat / sin hant getat (incomplete)

Jena $86 a$

HM IV, 8I9 १; JHSB I, I44 १, II, s s d

HM III, 92

8. Ich wiste gerne, wa bi man

die ritter solte erkennen,

ich sie vil... (incomplete)

Jena $92 \mathrm{~d}$

HM IV, $824 \uparrow$; JHSB I, I 55 १, II, 6I d

HM III, IOO

9. Kün' ich nu undersheiden wol zwene namen, wib und vrouwe, des wolt' ich mich vlizen Jena $97^{a}$ HM IV, 826 ; JHSB I, I62 १, II, 64 d HM III, IOS

10. Maria, muoter, meit und Kristes amme

Jena $83 a$

HM IV, 8 8 १ ; JHSB I, I $39 \uparrow$, II, 53 .

HM III, 88 
I I. Mich wundert, wie die wolken

vliegen tak und naht

Jena $88 \mathrm{~b}$

HM IV, 820 १; JHSB I, I48 १, II, $57 d$

HM III, 95

I . Sündige lust ist also sueze

Jena 95d

HM IV, 826 ; JHSB I, I60 १, II, 63 d

HM III, IO3

13. Swelich man ein werrer wesen wil Jena $93 \mathrm{C}$

HM IV, $824 \uparrow$; JHSB I, I 56 , II, 6I d;

zfdA LXXX (1943-44), 95 .

HM III, IOI

14. Vil sueze zarte minne

Jena $85 \mathrm{C}$

HM IV, 8 I 9 ; JHSB I, I43 १, II, s4 d

HM III, 9 I

Is. Vlize dich, mensche, an guotin wort Jena 9ic

HM IV, 822 ; JHSB I, I 53 \%, II, 60 d

HM III, 99

I6. Vür alliu wunder, diu nu sint, merket ein michel wunder

Jena $89 \mathrm{~d}$

HM IV, 82 I $\uparrow$; JHSB I, I 50 १, II, 58 d

HM III, 97

I 7. Zwiveler an deme gelouben, sich an Gotes wunder

Jena $90 \mathrm{c}$

HM IV, 822 ; JHSB I, I I I १, II, 59d

HM III, 98

- MÖNCH VON SALZBURG: See HERMANN DER MÖNCH -

28. MÜLICH VON PRAG

XIV C

TEBM 40; BMKH I 79

R. Batka, Die Lieder Mulichs von Prag. 1905 
I. Got, dine wunder manigfalt

„In Mülichs von Prage langer tone"

Colmar 78I

Runge, Colmar, $168 \uparrow$

2. Nu siht man aber beide

"Mülichs von Prage rey"

Colmar 40

Runge, Colmar, 249

Lang-Salmen 58 d, 19, facsimile

BMKH 199

See 9-3: Ascription to Mülich von Prag in APSB 29

BMKH I 85

29. MUSCATBLUT

- I439?

E. v. Groote, Die Lieder Muskatbluts. Köln, I 852.

I. Dung hab, der an begynne

"Muscatbl. nuwer ton"

Colmar 74; APSB 83

Runge, Colmar, 659

2. Esechiel der Prophet hel „Hoffton”

APSB $84 d$

3. Got vatter worcht on alle vorcht

"Muscatblutz alter ton"

Colmar 79

Runge, Colmar, $66 \uparrow$

30. NEIDHART VON REUENTHAL

I 190 ?-I 246 ?

Moriz Haupt und E. Wiessner, Neidharts Lieder. Leipzig, I923.

K. Ameln und W. Rössle, Tanzlieder Neidharts von Reuentbal. Jena, 1927.

Friedrich Gennrich, Melodien altdeutscher Lieder. 47 Melodien in bandscbriftlicher Fassung. Darmstadt, Musikwissenschaftliche Studienbibliothek, Heft 9, 1954.

Edmund Wiessner, Kommentar zu Neidharts Liedern. Leipzig, Hirzel, I954. Lieder von Neidhart (von Reuental), bearbeitet von Wolfgang 
Schmieder; Revision des Textes von Edmund Wiessner. Mit Reproduction der Handschriften. Wien, Universal, I930 (Denkmäler der Tonkunst in Österreich, LxxI) (abbrev.: DTO).

A. T. Hatto, R. J. Taylor, The Songs of Neidhart von Reuental. I7 Summer and winter songs, set to their original melodies, with translations and a musical and metrical cannon. Manchester, University Press, 1958. (abbrev.: HT).

F. Keinz, Neidbarts von Reuentbals Lieder. I9ro

E. Wiessner, Die Lieder Neidharts. Tübingen, Niemeyer, 1955. Texts included in this edition starred below.

See also Berlin 779. HM pages not given, since DTO LXXI replaces this edition.

* I. Allez, daz den sumer her mit vreuden was

Berlin 779, f. 215

DTO LXXI, $36 d$; HT $42 d$

Wehrli 348

*2. Blôzen wir den anger ligen sâhen

Berlin 779, f. I 59

DTO LXXI, 33 d ; HT I 4

3. Der mey der chumpt mit reicher wat

Vipiteno f. 49

DTO LXXI, 40 d

„Pseudo-Neidhart”

4. Der may gar wunnecleichen hat

Vipiteno f. 5 I

DTO LXXI, 40 d

"Pseudo-Neidhart"

5. Der may hat menig hercze hoch

erstaigett

Berlin 779, f. I60

DTO LXXI, 33 ; DAHA I, I 8 .

„Pseudo-Neidhart”

6. Der sumer kumpt mit reichem

geuden

Berlin 779, f. 201

DTO LXXI, 35 d

"Pseudo-Neidhart"

7. Der sumer kumpt mit reicher watt

Berlin 779, f. 198

DTO LXXI, 35

„Pseudo-Neidhart" 
8. Der sunnen (sumer) glast wenns von dem hymel scheynet

Berlin 779, f. 163 ; Wien 3344, f. 107 DTO LXxi, $33 d$; Gennrich, Melodien altd. Lieder, I I 9

„Pseudo-Neidhart"

9. Der swarcze dorn ist worden weis Berlin 779, f. I I I; Wien 3344, f. Ios DTO LXXI, 3 I $d$; Gennrich, Melodien altd. Lieder, 8

„Pseudo-Neidhart"

I0. Der vil lieben sumerzeit

Berlin 779, f. I7I; Wien 3344, f. 106 DTO LXXI, 34 d

"Pseudo-Neidhart"

I I. Der winter hat mit siben sachen uns verjagt

Berlin 779, f. 267

DTo LxxI, $39 d$; Gennrich, Melodien

altd. Lieder, ro $\uparrow$

„Pseudo-Neidhart”

* I 2. Des sumers und des winders beider veintschaft

Berlin 779, f. 217

DTO LXXI, $36 d$; HT $44 d$

13. Die liechten tag beginnen aber trüben

Berlin 779, f. 260

DTO LXXI, 39

„Pseudo-Neidhart”

* 14 . Do der liebe summer

Berlin 779, f. 228

DTO LXXI, 37 ; Gennrich, Melodien

altd. Lieder, 39 ; HT 20 d

I s. Do man den gumpel gampel sank

Wien 3344, f. 107

DTO LXXI, $40 \mathrm{~d}$; Gennrich, Melodien

altd. Lieder, I 1

„Pseudo-Neidhart” 
*I6. Ez gruonet wol diu heide

München, Staatsbibliothek, Cod. lat. 4660 (Carmina Burana)

Neidhart melody accompanying

„Annualis mea”?

17. Freut euch wolgemuten kindt

Berlin 779, f. I 5 ; Wien 3344, f. 108

DTO LXXI, 32 .

„Pseudo-Neidhart”

I8. Ich claghe de blomen und de wunnenlichen zit

Frankfurt f. 4

DTO LXXI, $4^{2}$

I9. Ich musz aber clagen gar von schulden

Berlin 779, f. 250

DTO LXXI, 38 d

"Pseudo-Neidhart"

20. Ich wen einen zagen

Berlin 779, f. 200

DTO LXXI, $35 d$; Gennrich, Melodien altd. Lieder 79

„Pseudo-Neidhart”

$*_{21}$. Ine gesach die heide

(Ich sah die haide)

Berlin 779, f. I 53

DTO LXXI, $33 d$; GTM III, 7a $d$; GFML 239 d Ameln-Rössle 6; Gennrich, Melodien altd. Lieder 2 १, Mbd. Liedkunst I9 १; HT I 2 d

22. Ir schawet an den lenczen gut

Berlin 779, f. 164

DTO LXXI, 34 d

„Pseudo-Neidhart”

23. Kinder ir habt einen wintter an der

handt

Berlin 779, f. 224

DTO LXXI, 37 d

„Pseudo-Neidhart" 
*24. Kint, bereitet iuch der sliten ûf daz is!

Berlin 779, f. 236

DTO LXXI, $37 d$; MGDM I, $166 d$; Gennrich, Melodien altd. Lieder $2 \%$;

HT I6 d GMMA 228 d

25. Mann hort nicht mer süssen schal

Berlin 779, f. 255

DTO LXXI, 39 d

„Pseudo-Neidhart"

26. May hat wunniglich entsprossen

Berlin 779, f. ${ }_{3} 6$

DTO LXXI, 31 d ; GFML $225 d$;

GMMA 227 ; Gennrich, Melodien altd.

Lieder I 29 ; Riemann 365 d

„Pseudo-Neidhart”

27. Mayenzeit one neidt

Berlin 779, f. I 49

DTO LXXI, $32 d$; GTM III, 7c d ;

GMMA $227 d$; HGEM 21 ; ;

Gennrich, Melodien altd. Lieder I 21 ;

Riemann 266

"Pseudo-Neidhart"

28. Meie, din liehter schin

Vipiteno f. 53

DTO LXXI, 4I $d$

29. Mey, du wunnenbernde zyt

Colmar 69

„Dyss ist h' Nytharcz ffrass"

Runge, Colmar, $62 \uparrow$, xviII d;

DTO LXXI, 42 d

See 30-30

30. Meye dein wunnewerde zeit

Vipiteno f. 59

DTO LXXI, 4I

"Pseudo-Neidhart"

3I. Mir is ummaten leyde

Frankfurt f. I

DTO LXXI, 4I $d$ 
*32. Mir ist von herzen leide Frankfurt f. I ; Berlin 779, f. 219 HM IV, 770, facsimile; DTO LXXI, 36 d ; Gennrich, Melodien altd. Lieder $4 \%$; HT 34 d

33. Nyemant soll sein trawren tragen lennger Berlin 779, f. I 78 ; Wien 3344, f. I04 DTO LXXI, 35 „Pseudo-Neidhart”

34. Niu horent wie sie all gemayne tihtent!

Vipiteno f. 57

DTO LXXI, 4I d (incomplete)

*35. Nu klag' ich die bluomen und die liehten sumerzît Berlin 779, f. 256 DTO LXXI, 39d; HT 28 d

36. Nun hat maye wunniglich beschonett Berlin 779, f. I 47r-v DTO LXXI, 32 •

- Nu var hin, vil ungedaner winter See 30-34 Incipit of text of 30-34

*37. Owê dirre nôt!

Berlin 779, f. 220 DTO LXXI, $36 d$; GFML $229 d$; Gennrich, Melodien altd. Lieder 2 १, Mbd. Liedkunst 20 १; HT 38 .

*38. Owê dirre sumerzît Berlin 779, f. 240 DTO LXXI, 38 ; Gennrich, Melodien altd. Lieder 6 ; н HT 24 d 
*39. Owê, lieber sumer, dîner liehten tage lange

Berlin 779, f. 238

DTO LXXI, $38 d$; HT $22 d$

- Owê liebe sumerzeit

See 30-38

*40. Owê summerzît

Berlin 779, f 222; Wien 3344, f. 106 DTO LXXI, $36 d$; HT 32 .

41. Owe winter, wie du hast beczwungen

Berlin 779, f. 258

DTO LXXI, 39 d

„Pseudo-Neidhart"

42. Seytt die lieben summertage

Berlin 779, f. 214

DTO LXXI, $35 \mathrm{~d}$; Gennrich, Melodien

altd. Lieder 9

„Pseudo-Neidhart”

*43. Si klagent, daz der winder

Berlin 779, f. 213

Dто LXXI, 35 ; ; Gennrich, Melodien

altd. Lieder 7 ; ; HT $_{40}^{\circ}$

*44. Sinc an, guldîn huon! ich gibe dir weize

Berlin 779, f. 234; Frankfurt f. 3

DTO LXXI, $37 d, 42 d$; GTM III, 7 b d ;

MGDM I, I6s d; Gennrich, Melodien

altd. Lieder 4 १, Mbd. Liedkunst I8 १;

HT I8-I9 d

Ameln-Rössle I6; Wehrli 342

*45. Sumer, dîner süezen weter

müezen wir uns ânen

Vipiteno f. 52

DTO LXXI, $40 d$; HT $30 d$ 
*46. Sumer unde winder

Frankfurt f. I

DTO LXXI, 4I $d$; HM IV, 770, facsimile;

HT $26 d$

- Sumers und des winders beider vientschaft: see $30-12$

47. Tochter spynn den rocken

Berlin 779, f. 168

DTO LXXI, 34 d

48. Uns ist komen ein liebe zeit

Vipiteno $f .48$

DTO LXXI, 40

"Pseudo-Neidhart"

49. Urlaub hab der winter

Berlin 779, f. I48; Vipiteno f. 47

DTO LXXI, $32 d, 4 \circ$

„Pseudo-Neidhart”

50. Was mir sender swäre

Berlin 779, f. 253

DTO LXXI, 38 d

„Pseudo-Neidhart"

- Wie schön wir den anger ligen sâhen

See 30-2

- Willekome eyn som... suze

Frankfurt, f. 2

DTO LXXI, 42 d

See $30-52$

5I. Willekomen mayenschein

Berlin 779, f. I4I

DTO LXXI, 3 I $d$; Parrish and Ohl,

Musical Masterpieces, I $3 d$; Riemann

264 d

„Pseudo-Neidhart"

52. Willekomen, sumerweter süeze

Frankfurt f. 2

DTO LXXI, 42

36 
- Winder...: see Winter...

53. Winter deine zeit

Berlin 779, f. $\mathrm{I} 37$

DTO LXXI, 3 I

„Pseudo-Neidhart"

54. Winter deiner kunfft der trawret sere

Berlin 779, f. 252

DTO LXXI, 38 d

„Pseudo-Neidhart"

*5. Winder, dîniu meil

Berlin 779, f. 23 I

DTO LXXI, $37 d$; HT $36 d$

56. Winter dir zu laide

Berlin 779, f. 166-67

DTо LXXI, $34 d$; Gennrich, Melodien

altd. Lieder 9 ,

„Pseudo-Neidhart”

57. Winder wie ist nu dein kraft

Berlin 779, f. I42

DTO LXXI, $32 d$; NOHM II, $256 d$;

DAHA I, I9 d; Gennrich, Melodien

altd. Lieder Io १; Riemann 265 d

„Pseudo-Neidhart”

- Wis wilkomen meienshin: $\mathrm{I}$

58. Wol dir liebe sumerzeit

Berlin 779, f. I 34

DTO LXXI, 3 I

„Pseudo-Neidhart”

59. Wol geczieret stet der plan

Berlin 779, f. 176 ; Wien 3344, f. 104

DTO LXXI, 34

„Pseudo-Neidhart”

60. Wolt ir hörn ein news geschiht

Vipiteno f. 54

DTO LXXI, 4I 


\section{I. OSWALD VON WOLKENSTEIN}

$$
\text { I } 377 \text { ?-I 430? }
$$

Oswald von Wolkenstein, Geistliche und weltliche Lieder bearbeitet, der Text von Josef Schatz, die Musik von Oswald Koller. DTo xvirr (Jg. $\mathrm{Ix}^{1}$ ) Wien, Artaria \& Co, I902. XXII, 233 pp. Single voice, nos. I-83; polyphonic, 84-г г6; Entwürfe und unvollständiges, I 17-24, * below; т marks texts, pp. I4-84.

Mss: A. Wien $2777.37 \times 27 \mathrm{~cm}$. 6Iff. xv c.

B. Innsbruck, Universitätsbibliothek. $49 \times 34 \mathrm{~cm} .48 \mathrm{ff} . \mathrm{xV}$ c.

C. Innsbruck, Museum Ferdinandeum, IV, c.I. 2 I $\times$ I $5 \mathrm{~cm}$. I I $5 \mathrm{ff}$. Paper. xv c. No music.

D. London, British Museum, Add. 24,946. 8 s ff. xv c. No music.

I. Ach Got, wär ich ein pilgerein

B 38

DTO I $39 \uparrow, 23 \mathrm{~T}$

2. Ach senleiches leiden

A 20, B 23

DTO I77 d, 2 I T

3. Ain anevank an götlich vorcht

A I, B I

DTO $139 \uparrow$, I40 $\uparrow$, S I T

$*_{4}$. Ain ellend schid durch zahers flins

A 37

DTO $206 \uparrow, 23 \mathrm{~T}$

$*_{5}$. Ain erens schatz an tadels ort

A 37

DTO $206 \uparrow, 23 \mathrm{~T}$

6. Ain graserin durch kuelen tau

A 36, в 36

DTO I77d, $22 \mathrm{I} \uparrow, 32 \mathrm{~T}$

7. Ain guet geporen edelman

A 47, B I9 DTO I $78 d$, I 79 १, 22T, facsimile, frontispiece

8. Ain jeterin, junk, frisch, frei, fruet

A 43, B 34, B 37

DTO I $40 \uparrow$, I $4 \mathrm{I} \uparrow, 32 \mathrm{~T}$

$3^{8}$ 
9. Ain mensch von achzen jaren klueg A 8 , в 26 DTO I4I $\uparrow$, I4 T

- Ain purger und ain hofman DTO $69 \mathrm{~T}$ Melody of 3 I-I6

Io. Ain rainklich weib durch jugent schön

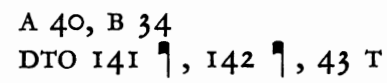

I I. Ain tunkle varb in occident
A 30, B I 6
DTO I42 $1,45 \mathrm{~T}$

12. a. Ave mueter, fraue, magtund maid b. Ave mueter küniginne B 44 DTO $205 d, 82$ T

I3. Bog depre mi was duster da

A Is

DTO I43 $1,23 \mathrm{~T}$

- Der himelfürst mich heut bewar DTO $63 \mathrm{~T}$

Melody of 3 I-59

14. Der mai mit lieber zal

A I9, B 22

DTO I $79 d, 3$ I T; DAHA $64 d$; J. Wolf, Gescbicbte der Mensuralnotation II, I40 \%, III, I 86 d ; zfdA LXXXII, I 20 d

- Der oben swebt und niden hebt DTO $54 \mathrm{~T}$

Melody of 31-59

I5. Der seines laids ergetet well sein

A 33

DTO $143 \uparrow, 37 \mathrm{~T}$ 
I6. Des grossen herren wunder

A 29, B I I

DTo I43 $9,47 \mathrm{~T}$

17. Des himmels trone empfärbet sich

A 34, B I 6

DTO I $82 d, \uparrow, 223 \uparrow, 26 \mathrm{~T}$

I8. Die minne füeget niemand

A 33, B 3 I

DTO I83 d, $29 \mathrm{~T}$

19. Do frayg amors

A 3 I, B 30

DTO I $44 \uparrow, 47 \mathrm{~T}$

20. Du armer mensch, lass dich dein sünd hie reuen ser

A 2, B 4

DTO I44 $9,56 \mathrm{~T}$

2r. Du ausserweltes schöns mein herz

A I 3, B 20

DTO I $83 d, 25 \mathrm{~T}$

22. Durch abenteuer tal und perg

A $42, \mathrm{~B} I 3$

DTо I $459,66 \mathrm{~T}$

23. Durch Barbarei, Arabia

A 49, B I9

DTO I $459,64 \mathrm{~T}$

- Durch toren weis so wird ich greis DTO $60 \mathrm{~T}$

Melody of 31-59

24. Erwach an, schrick, vil schönes weib

A 5 I, B I 7

DTO I46\%, I $8 \mathrm{~T}$

25. Es fuegt sich, da ich was von zehen jaren alt

A 9, B 8

DTO I 46 १, I 47 १, 4I T 
26. Es is ein alt gesprochen rat A IO DTO I47 १, 39 T; Forkel, Gescbichte der Musik

- Es komen neue mär greant DTO $73 \mathrm{~T}$

Melody of 3 I-85

- Es leucht durch graw die vein lasur DTO $34 \mathrm{~T}$

Melody of 3 I-I I

27. Es nahent gen der vasennacht

A 23, B 27

DTO I48 , 52 T

28. Es seust dort her von orient

A II, B IO

DTo I48 १, I49 १, Is T

- Freu dich, durchleuchtig junkfrau

A 56

DTO $83 \mathrm{~T}$

Melody of $3 \mathrm{I}-24$

29. Freu dich, du weltlich creatur

A 26

DTO I8s d, Is T

Wehrli 400

30. Freuntlicher plick

A 53, B 38

DTO I87d, I9 T

31. Fröleichen so well wir

A I7, B 2 I

DTO I $50 \uparrow, 20 \mathrm{~T}$

32. Fröleich geschrai so well wir machen, eachen

A 22, B 24

DTO I87 $d, 32$ T 
*33. Fröleich so will ich aber singen A 39, в 33 DTO 207 , 23 I 1, 49 T, facsimile, frontispiece

34. Frölich, zärtlich, lieplich und klälich, lustlich
A 32, B 24
DTO I $89 \mathrm{~d}$, I 8 T

36. Für allen schimpf, des ich vil sich

A $5 \mathrm{I}, \mathrm{B} 4 \mathrm{I}$

DTO I I $\uparrow, 38 \mathrm{~T}$

36. Gar wunnikleich hat si mein herz besessen

A 25, B 28

Dто I90 d, $2259,25 \mathrm{~T}$

37. Gelück und hail ain michel schar

A 23, B 27

DTO I I 1 , I4 T

38. Gesegent sei di frucht (Benedicite)
A $5, \mathrm{~B} 7$
DTO I $27,33 \mathrm{~T}$

39. Got gäb euch ainen gueten morgen A $4 \mathrm{I}, \mathrm{B} 34$

DTо I 537,49 T

40. Grasselick lif, war hef ick dick verloren

B 40

DTO I9I $d, 23 \mathrm{~T}$

4I. Her wirt, uns dürstet also sere

A 32, B 30; München Cgm 7 I 5

(fragment)

DTO I9I $d, 30 \mathrm{~T}$

42. Herz, muet, leib sel und was ich han

A 54, B 38

DTO I $54,26 \mathrm{~T}$

42 
43. Herz, prich! rich! mich

A 2 I, B 39

DTO $207 d, 22$ T

- Hör cristenhait

DTO $54 \mathrm{~T}$

Melody of $3 \mathrm{I}-3$

44. Hört zue was ellentleicher mär

(Compassio Beatae Virginis Mariae)

B 47

DTO I $54 \uparrow, 79 \mathrm{~T}$

45. Ich hab gehört durch mangen grans

A 44, B I 3

DTO I 547, I $59,67 \mathrm{~T}$

46. Ich hör sich manger freuen lat

A 38

DTO $206 \uparrow, 43 \mathrm{~T}$

47. Ich klag ain engel wunneklich, innerlich

B 44

DTO I92 $d, 82$ T (incomplete, two lines

only)

- Ich sich und hör

A 3 , B I 2

DTO $56 \mathrm{~T}$

Melody of 3 I-3

48. Ich spür ain luft aus küelem tuft A 6,87

Dто I5s 7, I 67, I6 T

Wehrli 402

- Ich spür ain tier

DTO $55 \mathrm{~T}$

Melody of 3 I-3

49. In Frankreich

A 7, в 6

DTO I 579,42 T

See 3 I-67 
50. In Oberland

A 57, B 45

DTO I $579,73 \mathrm{~T}$

- In Suria ain praiten hal DTO $35 \mathrm{~T}$

Melody of 3 I-I I

§I. Ir alten weib, ni freut euch mit den jungen

A I 2, B IO

DTо I 87, I $597,26 \mathrm{~T}$

52. Ir päbst, ir kaiser, du pauman

B 47

DTO I $591,79 \mathrm{~T}$

- Jenner besnaid Christ wirdikleich

DTO $35 \mathrm{~T}$

No melody

- Kain ellend tet mir nie so and DTO $62 \mathrm{~T}$

Melody of 3 I-59

- Kain freud mit klarem herzen DTO $55 \mathrm{~T}$

Melody of 3 I-I 6

- Keuschlich geporen DTO $33 \mathrm{~T}$

Melody of $3 \mathrm{I}-\mathrm{I} 7$

53. Kum liebster man!

B 44

DTO I93 $d, 45 \mathrm{~T}$

$*_{54}$. Lieb, dein verlangen

A 18, B 39

DTO $208 d, 22$ T

- Löbleicher got, gewaltikleicher DTO 65 T

Melody of 3I-3

44 
55. Mein herz das ist versert

A 30, B 28

DTO I93 $d, 25 \mathrm{~T}$

56. Mein herz jungt sich in hoher gail

A 30, B 29

DTO I94d, I9S $1,44 \mathrm{~T}$

57. Mein puel laist mir gesellschaft zwar

A 8, в 26

DTO $160 \%$, I $4 \mathrm{~T}$

58. Mein sünd und schuld euch priester klag

A $48, \mathrm{~B}$ I 7

DTO $160 \uparrow, 64 \mathrm{~T}$

59. Menschleichen Got, besniten schon

A 25, B I 4

DTO I60 १, $36 \mathrm{~T}$

- Mich fragt ain ritter angevar

D 85-89

DTO $75 \mathrm{~T}$

No melody

6o. Mich tröst ain adeleiche maid

A 30, B 33

DTO I95 d, $226 \uparrow, 44 \mathrm{~T}$

61. Mir dringt czwinget

München, Cgm 487I, p. I 35

DTO $84 \mathrm{~T}$

Rejected, DTO I 2

62. Mit günstlichem herzen wünsch ich dir

A 32, B 3 I

Dro $196 d, 46 \mathrm{~T}$

63. Nempt was der schönen plüede früede

B 43

DTO I6I $\uparrow, 24 \mathrm{~T}$ 
64. „Nu huss!” sprach der Michel von Wolkenstein

B 36

DTO I6I १, $47 \mathrm{~T}$

Wehrli 4 I6

65. $\mathrm{Nu}$ rue mit sorgen mein verporgenlicher schatz!

A 19

DTO I6I $9,17 \mathrm{~T}$

- O herzenlieber Nickel mein

DTO 28 T

Melody of $3 \mathrm{I}-78$

66. O Pfalzgraf Ludeweig

A 47, B 37

DTO I6 9 \%, $60 \mathrm{~T}$

- O rainer got DTо $62 \mathrm{~T}$

Melody of 3 I-67

67. O snöde welt

A 3, B S

DTO I62 १, $58 \mathrm{~T}$

See 3 I-49

68. O welt, o welt, ain freud der kranken mauer
A 3, B 3
DTO $1639,57 \mathrm{~T}$

69. O wunniklicher wolgezierter mai
A 52, B 4 I
DTо I64 १, $25 \mathrm{~T}$
GTM III, I7 $d$

70. $O$ wunnikliches paradis

A SI, B 40

DTO I64 १, $38 \mathrm{~T}$

- Rot weiss, ain frölich angesicht

B 37

DTO $44 \mathrm{~T}$

Melody of 3 I-8 
71. Sag an, herzlieb, nu was bedeutet uns so gar

A 17, B 22

Dто $196 d$, I 7 T

72. Senlich we mit langer zeit und weil vertreib

A 54, B 40

DTO I65 $9,45 \mathrm{~T}$

73. Sich manger freut das lange jar

B 4I

DTO I65 1, 7I T

74. Sim, Gredli, Gret, traut Gredelein

A 36, в 32

DTO $208 d, 2327,46 \mathrm{~T}$

Wehrli 410

75. Solt ich von sorgen werden greis

A 22, B 26

DTO I66 ๆ, $53 \mathrm{~T}$

76. Stand auff Maredel! Liebes Credel, zeuch

A I4, B 2 I

Copies in Wien, Cod. 19330 and 19224 DTO I97 $d$, 3 T

77. Sweig, guet gesell, schimpflichen lach

A 40, B 34

DTO I66 ๆ, $50 \mathrm{~T}$

78. Sweig still, gesell, dem ding ist recht

A 54

DTO $167 \uparrow, 28 \mathrm{~T}$

79. Treib her, treib überher

A 55, B 39

DTO $1677,29 \mathrm{~T}$

80. Tröstlicher hort, wer tröstet mich

A I 8, B 25

DTO $198 d$, I9 T 
- Und swig ich nu die lenge zwar DTO 8I T

Melody of 31-59

8 $\mathrm{I}$. Var, heng und lass, halt in der mass

A 7, B 8

DTO I67 १, I68 १, 2 I T

82. Vier hundert jar auff erd die gelten ainen tag

A 55, B 37

DTO I99d, 2 I T

83. Vil lieber grüesse süesse

A 44, B 19

DTO I $69 \uparrow, 27 \mathrm{~T}$, facsimile,

frontispiece

See 60-3

84. Von rechter lieb kraft

A 23, B 27

DTO 200 d, I9 T; J. Wolf, Geschichte

der Mensuralnotation, II, I 39 ๆ, III, I 84 d

85. Von trauren möcht ich werden taub B 42

DTO I $70 \uparrow, 70 \mathrm{~T}$

86. Von Wolkenstain wolt ich zu Cölen gueter laun

A 46, B I 8

DTO I7I $\uparrow, 6$ I T

87. Wach auff, mein hort! es leucht dort her

A 56, B 4I; Berlin 40,613, ff. 2, 7I; Rostock, Univ. Bibl., ms. phil. I00/2, f. Igr DTO $200 d$, I 7 T; SGMB 4I

Lang-Salmen $70 \mathrm{~d}:$ :Umbildung”;

Müller-Blattau, in Studien zur Musikgescbichte. Festschrift für Guido Adler (Wien, 1930), pp. 92-99 ; Gennrich, Melodien altd. Lieder I4,, 20 १, 2 I $\uparrow$, 241 
- Wach, menschlich tier DTO $52 \mathrm{~T}$

Melody of 3 I-3

- Weiss, rot mit praun verleucht DTO $25 \mathrm{~T}$

Melody of 3 I-55

- Wenn ich betracht DTO $53 \mathrm{~T}$

Melody of $3 \mathrm{I}-3$

88. Wenn ich mein krank vernunft närlicher sunder

A 5

DTO I7I $9,59 \mathrm{~T}$

89. Wer die augen wil verschüren mit den prenden

B 42

DTO $201 d, 72 \mathrm{~T}$

90. Wer hie umb dieser welte lust

B 48

DTO I72 १, $80 \mathrm{~T}$

9I. Wer ist die da durchleuchtet

A 4 , в 6

DTо $172 \uparrow, 173 \uparrow, 33 \mathrm{~T}$, facsimile, frontispiece

- Wer machen well den peutel ring DTO $37 \mathrm{~T}$

Melody of 31-23

92. Wes mich mein puel ie hat erfreut

A 22, B 25

DTO $173 \uparrow, 61 \mathrm{~T}$

- Wie vil ich sing und tichte DTO $68 \mathrm{~T}$

Melody of 3 I-I 6 
93. Wolauff als das zu himel sei (Gratias)

A 3, B 7

DTO I $29,33 \mathrm{~T}$

94. Wolauff, gesellen, an die vart

A 33

DTO I $74 \uparrow, 36 \mathrm{~T}$

95. Wolauff gesell! wer jagen well

A 24, B 23

DTO 202 d, 30 T; J. Wolf, Gescbicbte der Mensuralnotation II, I42 १, III, I89d

96. Wolauff und wacht

B 49

DTO $174 \uparrow, 82 \mathrm{~T}$

97. Wolauff, wir wellen slaffen

A 45, B 35

DTO 203 d, $30 \mathrm{~T}$

98. Wol auff, wolan! kind, weib und man

A 35, B 32

DTO $204 d, 230 \uparrow, 46 \mathrm{~T}$

99. Wol mich an we der lieben stund

A 24, B 28

DTO $175 \%$, IS T

100. Zergangen ist meins herzen we

A 48 , B 48

DTO $175 \uparrow, 176 \uparrow, 50 \mathrm{~T}$

Wehrli 412

- Zwar alte sünd pringt neues laid DTO $63 \mathrm{~T}$

Melody of 3 I-I I

IOI

A I 8 (no words)

DTO $209 d$, triplum

IO2.

A I 8 (no words)
DTO 209 , tenor

50 
32. GRAF PETER VON ARBERG XIV C

TEBM 70; BMKH I79

I. Ich wachter, ich solt wecken „Ein ander tagwyss Graf Peters von Arberg"

Colmar 826; Wien 2856, f. 214;

Wien suppl. 3344, f. 103

Runge, Colmar, I73 BMKH 578

2. Nu sterk uns Got in unser noit O starcker Got, al unser not (var.) "De passione Domini"

"Graff Peters grosse tagwyse" Trier, Stadtbibliothek, Hs. 724 (xv c.); Wien, Cod. membr. 7970 der K.K. Fideikommiss-bibliothek, f. 65a, 74a; Strassburg Hs. f. I I Ir; Colmar 828 Runge, Colmar, I73 १, I75 १, 176 I $77 \%$, facsimile, 174

E. Bohn, in Trierer Cäcilia, I 877, p. 83; Bäumker, Kath. d. Kirchlied, I, 452; K. Bartsch and F. M. Böhme, Germania XXV ( 1 880), 2 I० d; MGDM I, I 76 d; GTM III, I6 d; Schirmer, W. v.d. V. 696, facsimile BMKH 579; Zitzmann I 76

BMKH I 8 I

33. PETER VON REICHENBACH

I. Ey, froner wechter, wecke! „Dyss Peter von Richenbachs hort" Colmar 60

Runge, Colmar, 49 १, xvI

BMKH 23 I

Tagelied

2. Got, vatter, son, mit geistes fure

Colmar 62

Runge, Colmar, 53 ?

BMKH 234

Leich

See also 8-27 
BMKH I 84

\section{PETER VON SACHSEN}

I. Maria gnuchtig, zuchtig „Dyss ist ein barant ton her Peter von Sassen"

Donaueschingen 2 I 6a-2 I $7 \mathrm{~b}$; Colmar 38

Runge, Colmar, 22 ?

- POPPE: SEe BOPPE -

35. BARTHEL REGENBOGEN

Fl. I 300

BMKH I75; TEBM 42

Hermann Kaben, Studien zu dem Meistersinger Bartbel Regenbogen.

Diss. Greifswald, I930

I. Den vier elmenten gab zu sture „In Regenbogen gruntwysse”

Colmar 432 ; APsB 48

Runge, Colmar, I 7 \%

2. Die erste Epistel

„Dunner weise"

APSB 4I $d$

3. Du Got der herr

"In dem graen don Regenbogen"

Colmar 308; APSB 40, "Graben ton"

Runge, Colmar, is 7

4. Du wunnenberndes süss urspring

„Diss ist Regenbogen geticht

des heiligen cruczes Leich ie zwey

lieder in einem tone sint XXII töne"

Colmar 29r; Wien 270I, f. 22b-33b

Runge, Colmar, ro6-I 39 ; DTO XLI,

7I-77

HM III, 389-92

Leich

Regenbogen or Frauenlob?

See 8-55

5. Ein edel furste botten santte

"Regenbogen tagewyse"

Colmar 300; APSB 42

Runge, Colmar, I $4 \uparrow$

BMKH 385 
6. Ewiger, starcker, hoher, almechtiger Got

„Dyss ist die prueffwyse die ist Frauwenlobs und Regenbogen gemein daz iglich daryn tichtet" Colmar 244; APSB 43, "Briefe Weise” Runge, Colmar, ros?

7. Got vatter sprach zu Abraham "Im Regenbogen guldin ton"

Colmar 438 ; APSB 47

Runge, Colmar, i 8 ?

8. Ich singen ewig summersang „Hie fahent an die dryzehen reyen in der torenwise"

Colmar 283, 297

Runge, Colmar, i i 4

BMKH 3I-32

9. Maria, muter, reyne meyt, ein edel Gottes amme „In Regenbogen leyt don oder bluwend wyss" Colmar 442; APSB 39 Runge, Colmar i i 8 ?

ı०. Nun will ich dir sieben jahr dienen „Der meisterlich Hort, in vier gekroenten Toenen... Das vierte Gesetz, im langen Ton Regenbogens" (Other 3: 8-4I, 9-2, 25-9 HM IV, 935 ; Wagenseil 554

I I. Solt ich mit hohen fuersten geuden "In Regenbogen langen don" Colmar 333; APSB 38, „Der lange ton Regenbogen. Der 4 gekroenten ton einer"

Runge, Colmar i s $\uparrow$, i i 6 , facsimile See 35-10

Schirmer, W.v.d.V. I95

See 20-6 
Additional Tonen:

Braune ton, APSB 44

Süsse ton, APSB 46

Überlange ton, APSB 37

BMKH 163

$$
\begin{aligned}
& \text { 36. REINMAR VON BRENNENBERG } \\
& \text { XIII C }
\end{aligned}
$$

I. In dyser zyt mir grossen elend ist gegeben „In dem Brannenberger”

Colmar 672

Runge, Colmar is 89

2. Wol mich des tages dô mir alrêrst ist worden kunt

Kuhn-Reichert I59 d

37. REINMAR VON ZWETER

$$
\text { I 200?-I } 260
$$

TEBM 32, 43, 69; BMKH I $59-60$

G. Roethe, Die Gedichte Reinmars von Zweter. Mit einer Notenbeilage. Leipzig, I 887

DTO XLI

I. Almechtig schopfer aller creature "In Erenbotten spiegelwyse"

Colmar 721 ; Berlin 25, p. 25; APSB 70

Runge, Colmar I6I 9

BMKH $\lceil 24$

2. Der Prophet Malachias spricht „Erentpoden, Fürsten ton” APSB 68

- Dy mynne ist gut $9^{\text {th }}$ section of $37-4$

3. Es wont ein magt uff erden hie „Her Reymar von Zwetel fraw ern don"

Colmar 663; APSB 69 d,

„Erentpoden”; Donaueschingen 233

Runge, Colmar iss 1 
4. God und dyn ewen ewykit "Leich Reinmars von Zweter" Wien 2701, f. II DTO XLI, 62 gth section:

Dy minne ist gut

Wien 270I, f. I 2 DTO XLI, 63 d ; NOHM II, 257 d

5. Lucas im dritten der geschicht „Im Frauen Ehren Ton des Erenboten” Berlin 25, p. 27 HM IV, 922

6. Maria blüend rute „In Ernbotten schallwyse oder langer don"

Colmar 727

Runge, Colmar, I6I 1

7. „Salve regina, mater misercordie!” "In Rëmers sangwis von Zwetel” Donaueschingen 227

Runge, Colmar, I84 १, facsimile; Riemann $262 \eta$

38. REINOLT VON DER LIPPE

I. Min menscheit leider ist so krank Jena $45 \mathrm{~b}$ HM IV, 798 १; JHSB I, 79 १, II, 29 d HM III, 50

38a. MEISTER REYMAR

I. Daz eyme wol getzogenen man Münster fragment SIMG XII (I9IO-II), 500 d

39. RÖMER VON ZWICKAU, MARTIN - 5 April I483

I. Wem ein dugentsam weib bescheret ist auff erd „In der gesang weiss Romers" Berlin 25, p. 16; APSB 6r HM IV, 9259 Verfasser Lexikon questions ascription 
I. Nie man ze vruo sol prisen Jena $28 \mathrm{~b}$ HM IV, 790 १; JHSB I, 52 १, II, I9 d HM III, 3 I

4I. MEISTER RUMSLAND VON SACHSEN

BMKH 167

$$
2 / 2 \text { XIII C }
$$

I. Aller güete voller vlüete vlôz in gnâden strâmen

Jena $56 \mathrm{c}$

HM IV, 803 १; JHSB I, 97 १, II, 34 d Hм III, 6I; Wehrli 448

2. Das Gedeones wollen vlius intouwe Jena $5 \mathrm{rb}$ JHSB I, $89 \uparrow$, II, 32 d ; RMMA 235

3. Der künik Nabuchodonosor sach in eime troume Jena 5 Id HM IV, 80I १; JHSB I, 90 १, II, 33 d

4. Der wise heiden Cato, der nie touf gewan Jena $49 \mathrm{~b}$ HM IV, 800 १; JHSB I, 85 १, II, 3 I d HM III, 53

5. Got herre almechtich Jena $58 \mathrm{c}-59 \mathrm{a}$ HM IV, 803 ; J JHSB I, IOI १, II, 36 d HM III, 63

6. Got in vier elementen sich erscheint Jena $47 \mathrm{C}$ HM IV, 799 १; JHSB I, 82 १, Ir, 30 d 
7. Herre und meister, schepfer min Jena $59 \mathrm{~d}$ HM IV, 804 १; JHSB I, IO2 १, II, 37 」 HM III, 65

8. Man fraget hoch, wo Got behuset were „Im geswinden ton meinster Rumsland Etlich sprechen Wolframs." A later band bas added: „hort dem Frawenlob $\mathrm{zu}$ ist sin thon"

Colmar 776

Runge, Colmar, 1669 BMKH 547

9. Ob aller minne minnen kraft Jena $55 \mathrm{~b}$ HM IV, 802 १; JHSB I, 95 १, II, 33 d Wehrli 447

Record: Anthologie Sonore, 18

Iо. Untriuwe slichet, also ein mus Jena $62 \mathrm{~b}$ HM IV, 80 ○ १; JHSB I, IO6 १, II, 38 d; GTM III, I 2 d HM III, 67

\section{RUMSLAND VON SCHWABEN}

I. Ich han nach wane dikkegelobet Jena 62d-63a HM IV, $806 \uparrow$; JHSB I, IO7 १, II, 38 d HM III, 68

43. DER TUGENDHAFTE SCHREIBER XIII C

BMKH I 58

I. Ein wyser man het einen son „In der grüsswyse dez tugenthafften Schrybers" Colmar 742 Runge, Colmar, 164 нм I, 364, under Winsbeke 
I. Swer ritters namen welle enpfan Jena $43 \mathrm{~d}$

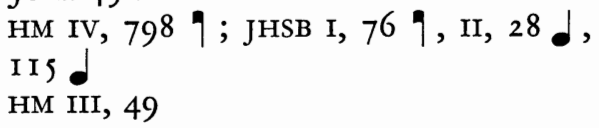

\section{SPERVOGEL \\ XII C}

I. Swâ ein vriunt dem andern

vriunde bî gestat

Jena 29a

HM IV, 790 ; JHSB I, 54 १, II, 20 d;

DAHA I, I 8 ; Riemann $260 d$

Vogt, Des Minnesangs Frübling, 24

- Swer suochet rât und volget des, der habe danc

Jena

MGDM I, I5 6

Vogt, Des Minnesangs Frübling, 20

46. DER ALTE STOLLE

TEBM 46, 7I; BMKH I 64

W. Seydel, Meister Stolle nach der Jenaer Hs. Diss. Leipzig, I 893

I. Christus saget das himelreich „In der Alment des Stollen”

Berlin 25, p. I 8

HM IV, 921 ?

2. Ich wil dich bitten, milter Got, wan du durch uns den tot

„In der alment des alten Stollen”

Colmar 706; APSB 65

Runge, Colmar, I 59 ; JHSB II, I 98 .

BMKH SII

3. Lob aller engel unde lob der rechten kristenheit

„Meyster Stolle”

Jena $2 \mathrm{a}$

HM IV, 775 ; JHSB I, 2 १, II, I d

HM III, 3 
4. "Blutton", АPSB 67

See 46-3

47. DER JUNGE STOLLE

TEBM 7I; BMKH I 68

I. Esaias der thut beschreiben

„Hohe ton"

APSB 66 ; Berlin 25, p. 2 I (no words)

2. Schaffzabel wart vor Troy herdacht

„Dyss ist des jungen Stollen getichte”

Colmar 7 I 9

Runge, Colmar, I60

HM II, 375; BMKH 523

\section{SUCHENSINN}

XIV C

TEBM 7I; BMKH I8 I

Emil Pflug, Suchensinn und seine Dichtungen. Breslau, I 908.

I. Ich kam uff einen anger wyt

„Dyss ist in meister Suchensin tone"

Colmar 8 2

Runge, Colmar, $172 \uparrow$;

zfdA Lxxx (1943-44), 97

BMKH 562

2. Plutarchus beschreibet mit Neron

„Im... des Suchsimsinn"

Berlin 24, f. I 57 r

49. TANNH ÄUSER

I 20 S ? - I 270

BMKH I62; TEBM 40

A. Oehlke, $Z u$ Tannhäusers Leben und Dichten. Diss. Königsberg, I 890. Johannes Siebert, Der Dichter Tannbäuser, Leben, Gedicbte, Sage. I934. Margarete Lang, Tannbäuser. Leipzig, 1936.

Tannbäuserlied

„Welle grosse wunder schauen will,” Schweizer Volkslied, HM Iv, 936 d;

Franz M. Böhme, Altdeutsches Liederbuch. Leipzig, Breitkopf u. Härtel, I877, p. 86 . , ,Wolt wir aber haben an”, Böhme 82 \%. 
I. Ez ist hiute ein wunniklicher tak

Jena $42 \mathrm{~d}$

Hм IV, $797 \uparrow$; JHSB I, 74 १, II, 27 d;

NOHM II, 254 d

HM III, 48

2. Gelückes wer mir not

„In Tanhusers heuptton oder guldin tone"

Colmar 785 ; Berlin 25, p. 359

Runge, Colmar I69 9; JHSB II, 200 d

- Ich lobe ein wip, diu ist noch bezzer danne guot

German text to 49-4

HM II, 85-87

3. Mir tet gar wol ein lieber wan

„Des Danhusers lude leich"

Colmar 72

Runge, Colmar, 641

BMKH 245-5 I

4. Syon egredere nunc de cubilibus

München 5539, ff. I6I-67

Spanke, zfMw XIV(I931-32), 385-97 d;

zfdA LXIX (1932), 49-70; Hugo Kuhn, Minnesangs Wende (Tübingen, Niemeyer, I952), 164-70, facsimile

German text: Ich lobe... above

5. „Hofton”, APSB 97

50. ULRICH VON WINTERSTETTEN

Fl. I 240-I 280

J. Minor, Die Leiche und Lieder des Schenken Ulrich von Winterstetten. I 882

A. Selge, Studien über Ulrich von Winterstetten (German Studien LXXI, 1929)

I. Diu minne stoeret unde toeret

mich ân allen wân

Schreibers fragment (lost)

Facsimile in Kuhn, Minnesangs Wende (see 49-4), I 64; Schreiber, Taschenbuch für Geschicbte und:Altertum in Süddeutscbland. KLD 503-508, Leich IV, "Swer die wunne, of which Diu minne... is line 53 (p. 505) 
- Ich möhte ersterben sol min werben niht erschiezen mir

Line 5 I of Leich IV Kuhn-Reichert 160

5I. DER UNGELEHRTE

Fl. I300?

TEBM 46, 7I; BMKH 166

I. Sang ist ein wyse meinsterschaft "In dem Ungelarten"

Colmar 750

Runge, Colmar, I 649

52. DER UNVERZAGTE

2/2 XIII C

I. Der künik Ruodolf minnet Got

und ist an triuwen staete

Jena 4 od

HM IV, 795 १, 860f $d$; MGDM I, I68 $d$;

RMMA $234 d$; GTM III, I I $d$; JHSB

I, $70 \%$, II, $26 \%$; GMMA 222 ;

HGEM $2 \mathrm{I} d$; Moser, Minnesang und

Volkslied (Leipzig, I929), I 7

HM III, 45

Record: Decca 20158

2. Ez ist ein lobeliche kunst

Jena $40 a$

HM IV, 795 १; JHSB I, $69 \uparrow$, II, $25 d$;

GMMA 222 d

HM III, 44

3. Junger man von zweinzik jaren

Jena $39 \mathrm{~b}$

HM IV, 794 १; JHSB I, $68 \uparrow$, II, $24 d$;

GFML I 92 d

HM III, 43 
Verfasser Lexikon IV, 682

\section{VEGEVIUR}

I. Maria, muoter unde maghet

Basel N.J. 3, nr. I45

Spruchstrophe

54. WALTHER VON DER VOGELWEIDE

$$
\text { I } 170 \text { ? -I } 230 \text { ? }
$$

BMKH I 6

Die Gedichte Walthers von der Vogelweide, zwölfte Ausgabe, (Karl Lachmann) ed. Carl v. Kraus. Berlin, De Gruyter, I959.

Walther von der Vogelweide, Sprüche, Lieder, der Leich. Berlin, Tempel, I955.

Walther von der Vogelweide, Die Gedichte. Berlin, De Gruyter, I955. Friedrich Maurer, Die Lieder Walthers von der Vogelweide. I, Die religiösen und die politischen Lieder, I955. II. Die Liebeslieder. Tübingen, Niemeyer, I955-56.

Karl-Heinz Schirmer, Die Strophik Walthers von der Vogelweide. Halle, Niemeyer, 1956.

Kienast, „Walthers ältester Spruch im Reichston," Gymnasium LVIr, 20I ff. R. Wustmann, „Die Hofweise Walthers von der Vogelweide,” Liliencron Festscbrift, pp. 440-63. Leipzig, I910.

Friedrich Pfaff, Der Minnesang des I 2. bis 14. Jabrbunderts. Abteilung 2.

Friedrich Gennrich, "Zur Liedkunst Walthers von der Vogelweide," zfdA Lxxxv (1954-55), 203-09.

Fr. Pfeiffer, „Zwei Lieder Walthers von der Vogelweide," Germania II (I 857 ), $470 \mathrm{ff}$.

K. Plenio, „Die Überlieferung Waltherscher Melodien,” PB Beitr. XLIr, 479-90.

Carl von Kraus, Walther von der Vogelweide, Untersuchungen. Berlin, De Gruyter, I935.

Rudolph Wustmann, „Walthers Palaestinalied," SIMG XIII (I9II-I 2), 247-50.

C. Bützler, Untersucbungen $z^{u}$ den Melodien Walthers von der Vogelweide (Deutsche Arbeiten der Universität Köln, Nr. I 2). Jena, I940, Pp. I I 2. Friedrich Gennrich, „Melodien Walthers von der Vogelweide," zfdA LXXXI (I 942), 24-28.

J. A. Huisman, Neue Wege zur dicbterischen und musikalischen Technik Walther von der Vogelweide (Studia Litteraria Rheno-Traiectina, I). Utrecht, I950.

Wolfgang Mohr, „Zu Walthers 'Hofweise' und 'Feinem Ton”, zfdA LXXXV (I $954-55)$, 38-43. 
I. Am dreizehenden sein Mathei sagt Christus

„In Creuz Ton Walter von der Vogelweid"

Berlin 25, p. I 2

2. Das ein und sechzigste Caputt Esaie „Feine ton"

APSB 58

Wustmann, op. cit.; Bützler 66 १

Ascription: see 54-I I

- Allerest leb' ich...: see $*_{2 \mathrm{I}}$, below

3. Die trinitat gedryet

"In her Walthers guldin wyse"

Colmar 736

Runge, Colmar, $\mathrm{I}_{3} \uparrow$; Bützler so ๆ, facsimile

4. Diu menscheit muoz verderben See 54-I 2, 26

5. Diu welt was gelf, rot unde blâu Melody of Gautier d'Espinal, Amours et bone volentés zfdA LXXIX, 46 .

*6. Friuntlichen lac ein ritter vil gemeit "Walthers Tagelied"

Maurer II, I 35 d; zfdA LxxIx, 38 d; Gennrich, Mbd. Liedkunst $12 \eta$

Schirmer 200

7. Frô Welt, ir sult dem wirte sagen Melody of Blondel de Nesle, Onques nus hom ne chanta zfdA Lxxix, 47 ; Gennrich, Mbd. Liedkunst 179

8. Got gît ze künege swener wil Bützler 68 ๆ

See $54^{-2}$ 
*9. Her babest, ich mac wol genessen „Der Ottenton”

Aarburg, op. cit.; APSB 58 (p. I75a) GTM III, Ga d Bützler 731 ; Gennrich, Mbd. Liedkunst I 3 १; contrafactum: APSB 58 (p. 175a)

Wustmann 444; Maurer I, so d

See 54-10

* I0. Her keiser, sit ir willekomen

(March, I 2 I 2)

„Der Ottenton”

Maurer I, $49 d$; zfdA LxxIx, 39d

Wehrli 218

See 54-9

I I. Hiehört wiein der ApostelGeschicht „In des $\mathrm{H}$ : Johannis Walters tön 3.

Ein Landherr"

"Lange ton"

APSB $56 d$ (p. 172b); Bützler 64, facsi-

mile, $95 \%$, under ,unechte Melodien"

I2. Ich freu mich des das mir

Ascription: see 54-I I

"Creuzton”

APSB $57 d$ (p. I 74a); Bützler 759

See 54-4

$*_{13}$. Ich horte ein wazzer diezen

„Der Reichston”

Maurer I, 28; zfdA LxxIX, 43 d

Kraus IO

*I4. Ich saz uf eime steine ( $c a$. I I 98 -I 20I)

„Der Reichston”

Maurer I, 27

Wehrli 208

I 5. Maneger klaget die schoenen zît

Bützler 62 d

See 54-3

64 
16. Mary, du bist daz bernde ryss „Her Walthers von der Vogelweyde hoffwyse oder wendelwys"

Colmar 734

Runge, Colmar, I62 १; zfdA Lxxix, 34 d; Bützler 40 १, facsimile, 46-47 १ HM I, $257-60$

See $54-24,28$

$*_{17}$. Mir hat ein lieht von Franken

(ca. 1 207-08)

Münster fragment

SIMG XII (I9IO-II), $500 d$;

MGDM I, I6I d Bützler 8 \%, r4-Is d; zfdA LxxIx, 29 ; Maurer, I, 43

Tempel edn., I7; DeGruyter, I45

Maurer: „Der zweite Philippston”

*18. Mir hat her Gerhart Atze ein pfert erschozzen $z$ Isenache

(before 1 207)

„Der erste Atzeton”

Maurer, I, 4I $d$; zfdA LxxIx, $42 d$

* I9. Mit saelden müeze ich hiute ufsten (ca. I 206)

„Der Wiener hofton”

Maurer, I, 33 d

See 54-28

$*_{20}$. Muget ir schouwen waz dem meien Melody of Gautier d'Espinal, Quant je voi l'erbe menue; Carmina Burana, nr. Is I, I69 (f. 6Ir): see Bützler Ios Maurer II, $57 d$; zfdA LxxIx, 47

* $_{\text {2 I. }} \mathrm{Nu}$ alerst leb' ich mir werde „Palestina Lied” ( 1228 )

Münster fragment

SIMG XII (I9IO-I I), $499 d$; MGDM I, $162 d$; SGMB $6 d$; RMMA $234 d$;

NoHM II, 253 d ; DTO XLI, $87 d$; Adler, 
Handbuch der Musikgeschichte I, 2, 204 d; GFML $247 d$; GTM III, 6b $d$; GMMA 2I9 d DAHA I, I $8 d$; SIMG XIII (I9III2), 247-50; HGEM 20 ; ;

Z.f.d. Bildung II (1926), 630; Huisman I47; Maurer I, I 5 d ; H. Husmann, Die Musikforschung vI (1953), I7 d; zfmw vIr, $98 \mathrm{~d}$; zfdA LxxIx, $32 \mathrm{~d}$; A. A. Abert, Musikforschung I (I948), 103 ff.; Gennrich, Mbd. Liedkunst I 6 ; Bützler 26 १, $28 d, 29 d, 34 d$; Melody of Jaufré Rudel, Lanquan li jorn son long en mai: see GTM $5 \mathrm{I}$

Tempel 78; De Gruyter 271

Records:Decca 20158; Anthologie Sonore 18

*22. Philippe, künic here „Der zweite Philippston” GTM III, 6c d ; Z. f.d. Bildung II (1926), 63 I : Maurer I, $44 d$; Gennrich, Mbd. Liedkunst I4 9

Studi Medievali xVII (I95 I), 7I-85

See 54-17

$*_{23}$. Sie wunderwol gemachet wip Kremsmünster, Stiftsbibliothek, Ms. I 27, VII, I 8, f. I 3 or Maurer II, 96 .

24. Swer houbet sünde unt schande zfdA Lxxix, $3^{6} d$; Bützler 479 See 54-16

25. Uns hât der winter geschât über al Melody of Moniot de Paris, Quant voi ces prés florir et verdoier zfdA Lxxix, 46 d

26. Vil süeze waere minne

„Walters Kreuzlied”

Bützler 79 d

See 54-4, 54-I 2 
*27. Vil wol gelobter Got, wie

selten ich dich prîse! (I 2 I 2-I3)

„Die könig Friedrichston”

Münster fragment

Maurer I, $54 d$; SIMG XII (I9IO-II), 499 d ; MGDM I, I6I d ; SGMB 7 d;

Bützler $16 \%, 23$.

BMKH 536

Line 8: Wie solte ich den geminnen

$*_{2} 8$. Waz wunders in der werlte vert

„Der Wiener Hofton”

GTM III, 6d d ; Bützler 40 1, 49 d ;

Mantuani, Gescbicbte der Musik

in Wien 1, 299; Gennrich, Mbd.

Liedkunst I I $\uparrow$

Maurer I, 35

See 54-16, 24

- Wie solte ich den geminnen: 54-27

*29. Wol mich der stunde, daz ich sie erkunde

Melody of Bernart de Ventadorn, Can vei la flor, l'erba e la folha

Maurer II, 89 $d$; zfdA LxxIx, 45 d;

Gennrich, Mbd. Liedkunst I6 \% Mo-

ser, zfMW XVI (I934), I 5 of; zfMW

LXxxvir, 43.

See also 60-2

- DER WARTBURG KRIEG -

See IO-I, I8-I, 57-I

and Verfasser Lexikon IV, 843-64; HM II, I-I9, III, I70-82

55. BRUDER WERNER

XIII C

A. Schönbach, Die Sprüche Bruder Werner (Sitzungsberichte der Akademie der Wissenschaft zu Wien, Phil.-his. Klasse, 1904, nr. 7; 1905, nr. 6)

I. Ich buwe ein hus, da inne wil gesinde wesen

Jena $14 \mathrm{~d}$

HM IV, $778 \uparrow$; JHSB I, $27 \uparrow$, II, 6 d

HM III, I9 
2. Ich weiz der herren manigen ob ich het ir eines guot Jena I $2 \mathrm{C}$ JHSB I, 23 १, II, 4 d ; HM IV, 777

3. $\mathrm{Nu}$ merket, wa ein blinder get Jena $14 a$ HM IV, $778 \uparrow ;$ JHSB I, 26 १, Ir, 5 d

4. Nu schouwet an den sumer guot Jena $9 \mathrm{~b}$ HM IV, 776 १; JHSB I, I7 १, II, 3 d, I I 7 d

5. Swer sich mit vremden liuten wil Jena isd HM IV, 779 १; JHSB I, 29 १, II, 7 」

6. Wir lan die pfaffen sin vertan Jena $7 \mathrm{~d}$ HM IV, $775 \uparrow$; JHSB I, I 2 १, II, 2 d HM III, I I

$$
\begin{aligned}
& \text { 56. WIZLAV (III, FÜRST) VON R ÜGEN } \\
& \text { I265?-I325 }
\end{aligned}
$$

Ludwig Ettmüller, Wiqlavs Sprüche und Lieder (Bibl. der d. nat. Literatur XXXIII), I 8 \%2.

E. Gülzow, Des Fürsten Wiqlaw von Rügen Minnelieder und Sprüche, 1922. A. Dölling, Die Lieder Wizlaws III von Rügen, klanglich und musikalisch untersucbt. Diss. Leipzig, 1926.

Friedrich Gennrich, „Zu den Melodien Wizlavs von Rügen," zfdA Lxxx (1943-44), 86-102.

R. J. Taylor, „A Song by Prince Wizlav of Rügen,” Modern Language Review XLVI (195I), 3I-37.

I. Der herbest kumt uns riche genuoch Jena 8 od HM IV, $817 \uparrow ;$ JHSB I, I $35 \uparrow$, II, 52 d

HM III, 85; PMDL I, 249

2. Der Ungelarte heft gemaket Jena $75 \mathrm{c}$ HM IV, 8I I १; JHSB I, I 27 १, II, 45 d; Lang-Salmen $64 d$ HM III, 8 I 
3. Der walt unde anger lit gebreit

Jena $79 a$

HM IV, 8 I 59 ; JHSB I, I 32 , II, 5० d;

MGDM I, I 7 I d Riemann 268 d

HM III, 84

4. Diu erde ist entslozzen

Jena $77 \mathrm{~d}$

HM IV, 8I $3 \uparrow$; JHSB I, I 30 १, II, $48 \mathrm{~d}$;

Riemann 268

HM III, 82

5. Diu vogelin

Jena $79 d$

HM IV, 8 I 6 ; JHSB I, I $34 \uparrow$, II, s I d;

GFML 207

HM III, 84

6. Ich parrere dich durch mine trowe Jena 75 a

HM IV, 8 8 10 १, 860f d; JHSB I, I $27 \uparrow$, II, $44 \mathrm{~d}$; NOHM II, $253 \mathrm{~d}$; Lang-Salmen 62

HM III, 8I

7. Ich warne dich, vil junger man gezarte

Jena $77 a$

HM IV, 8 I 3 ; JHSB I, I 29. १, II, 47 d

HM III, 82

Incomplete

8. List du in der minne dro

Jena 77 a

HM IV, 8 I 2 १; JHSB I, I 29 ๆ, II, 47 d ;

GTM III, I sd d Riemann, $27 \mathrm{I} d$;

GFML 239d ; GMMA $223 d$

HM III, 8 I -82

9. Löuvere risen von den bômen

hin to dal

Jena $80 \mathrm{~b}$

HM IV, 8 I 6 \%, 86of d ; GTM III, I sc d;

F. Gennrich, „Liedkontrafactur

in mhd. und ahd. Zeit," $z f d A$

LXXXII (1948), I 34 d ;

Riemann, 269d; JHSB I, I 34 \%, II, 5 I

HM III, 8 s 
ı. Manik schimpfet uf sin eigen zil Jena $76 \mathrm{c}$ HM IV, 8 I 2 १; JHSB I, I 28 १, Ir, 46 d ; Lang-Salmen 66 HM III, 8I

II. Meije schoene, kum jo zuo Jena $78 d$ HM IV, 8I 5 ; J JHSB I, I 32 १, II, 49 d HM III, 83 ; PMDL I, 248

I2. Menschen kind, denket dar an Jena $73 a$ Hм IV, 809 १; JHSB I, I 24 १, II, 42 d HM III, 78

I 3. Nach der senenden klage muoz ich singhen Jena $76 \mathrm{~b}$ HM IV, 8 I I $\uparrow ;$ zfdA Lxxx, $87 d ; 94 d$ GTM III, I 5 a $d$; JHSB I, I 28 ๆ, II, 46 d; Gennrich, Mbd. Liedkunst 2I १; MLR XLVI, 3I 9-37 d

HM III, 8 I

I4. O we, ich han gedacht Jena $77 \mathrm{c}$ HM IV, 8 13 १; JHSB I, I 30 १, II, 47 d; GMMA $218 d$; GTM III, I $5 \mathrm{~b} d$; MGDM I, I70 d HGEM 20 d ; Gennrich, $M b d$. Liedkunst $2 \mathrm{I} 1$

HM III, 82

Record: Decca 20I58

I 5 . Sage an, du boser man Jena $74 \mathrm{c}$ HM IV, 809 १; JHSB I, I 26 १, II, 43 d HM III, 80

- We, ich han gedacht: $O$ we... 
I6. Wol dan, her meije, ich gibe iuch des die hulde

Jena $79 \mathrm{c}$

HM IV, 8I 9 ; JHSB I, I 33 १, II, 5O d

HM III, 84

I7. Wol uf, ir stolzen helde

Jena 78 a

HM IV, 8 I 4 १; JHSB I, I 3 I $\uparrow$, II, 48 d;

MGDM I, I69 d

HM III, 83 ; PMDL I, 246

57. WOLFRAM VON ESCHENBACH

Fl. I 200-I 220

BMKH I57

Wolfram von Eschenbach, ed. Albert Leitzmann. Halle, Niemeyer, 1926. Lieder: Heft v, I79-87.

I. Als Christus wandeln thete „Wolff Rones eines Ritter"

"Kreuzton"

APSB 50 d

2. Als Gott saget zu Abraham

„Wolff Rones eines Ritter"

"Lange ton"

APSB 49 d ; MGDM I, 316 d

3. Do man dem edelen sin gezelt

"Im swarzen don"

„Kriek von Wartberk"

Jena $127 \mathrm{~d}$

HM IV, $844 \uparrow$; JHSB I, 22 I $\uparrow$, II, 84 d

HM III, I 7 I

4. Hoseas der heylig Prophet

"Wolff Rones eines Ritter"

"Flamm weise"

APSB 53 d

5. Jamer ist mir entsprungen

Wien, Titurel Hs. 40

HM IV, 774, facsimile; MGDM I, I63 d 
6. Jeremias weissaget

"Wolff Rones eines Ritter"

"Vergolten ton"

APSB 54

Nuremberg $784 d, 792 d$, under

APSB 54

7. Matheus schreibt am achten

Christus drat in ein schiff

"In der Honn' weiss Wolframs"

Berlin 25, p.I

HM IV, 921 ?

8. Was sol ein keyser one recht

„In Wolframs guldin tone von Eschel-

bach"

Colmar 730

Runge, Colmar I62 1 ; APSB 52

HM II, 260; BMKH 535

9. „Hohe weise”

APSB 55

Iо. „Kurze ton”

APSB SI

58. MEISTER ZILIES VON SAYN

I. Ein kupfer so verguldet was, daz ez gar guldin shein

Jena 2 ra

HM IV, $783 \uparrow$; JHSB I, 39 १, II, II

HM III, 25

2. So wol dem hobe, da man triuwe an hoher wirde hat Jena $20 \mathrm{C}$ HM IV, 782 ; JHSB I, 38 १, II, IO d; GTM III, IO HM III, 25 
59. ZWINGER

$2 / 2$ XIV C

BMKH I 82

I. Adam und Even schuld wart hart uns armen

"In des Zwingers rotten don, sin hort”

Colmar 79; APSB 86

Runge, Colmar, 66

2. „Hofton”, APSB 85 .

60. ANONYMOUS

I. Ich han vor lorin den lybyster bulen myn

Wien 2701

DTO XLI, 62 d

2. Ich setze minen vuz (fuoz)

Magdeburger Archiv

Berlin 981

HM IV, 773, facsimile; NOHM II, $25 \mathrm{~s} d$;

Wolf, Handbuch der Notationskunde

I, $7^{6} \mathrm{~d}$, facsimile; Wolf, Musikalische Scbrifttafeln, pl. 2I, facsimile;

Kuhn-Reichert I 57 d

KLD I, 277

3. Man sieht lawber tawer

Vipiteno 35b-36a

Zingerle, Wien Sitzungsberichte... LIV, $324 \mathrm{ff}$; DTO XVIII, 108

Umdichtung of Jam entrena plena: see $3 \mathrm{I}-83^{\circ}$

4. ...sin hanne genommen

Münster fragment

SIMG XII, $49^{8} d$; Bützler 99-IOI

„Neue Ton" of Walther von der Vo-

gelweide? See Bützler 99-102

5. Rôsen ûf der heide mit leide

Erlangen I $3 b$

Kuhn-Reichert I 57 


\section{Wat den bin ic? ein spilemen}

Karl Bartsch's XIII c. ms.

fragment (lost?)

Erk-Böhme, Deutsche Liederbort

(Leipzig, I925), II, 189, 376:

„mit zweifelhaft übertragener

Melodie", Lang-Salmen, 123.

Lang-Salmen 72

\section{I. MINNESINGER, TROUBADOURS, TROUVÈRES}

An increasing attention to musical and literary relationships between these three has produced, among others, the groups of related poems below, together with the following bibliography:

Ursula Aarburg, op. cit. supra; K. Bartsch, in Germania I (1856), 480-82; O. Schultz-Gora, zfdA XxI (1887), I 85-89; C. von Kraus, Untersuchungen; F. Michel, Heinrich von Morungen und die Troubadours (Quellen und Forschungen xxxVIII), I88०; F. Gennrich, „Der deutsche Minnesang in seinem Verhältnis zur Troubadour- und Trouvère-Kunst", Z.f.d. Bildung II (1926), 536-68, 622- 32; F. Gennrich, „Sieben Melodien zu mittelhochdeutschen Minneliedern," zfMW VII (1924-25), 65-98; H. Spanke, „Romanische und mittellateinische Formen in der Metrik von Minnesangs Frühling," zfrp XLIX (1929), 190-235; H. Spanke, Beqiebungen qwischen romanischer und mittellateinischer Lyrik (Abhandlungen der Göttinger Ges. d. Wiss., Phil.-Hist. Klasse, 3. Folge, Nr. I8), I936; H. J. Moser, „Zu Ventadorns Melodien," zfMw xvi (1934), I 5 off.

István Frank, Trowvères und Minnesänger. 2 vols., Saarbrücken, I952, 1956. Vol. I. Recueil de Textes; II, Kritische Ausgaben der Weisen... von Wendelin Müller-Blattau.

See also 54, Walther von der Vogelweide.

\section{ALBRECHT VON JOHANSDORF}

\section{Ich vant âne huote}

Un Marquis, Dona a vos me coman

Frank I, 86-9I, no melody

\section{BERNGER VON HORHEIM}

2. Mir ist alle zît als ich vliegende var

Anon., Puis ke li mal k'Amours me font sentir

Bertran de Born, Miez sirventes vueilh far dels reis amdos

Frank 1, 92-97, II, 91 9

3. Nu lange ich mit sange die zît hân gekündet

Gace Brulé, Ne puis faillir a bone chançon fere

Frank I, IO8-I I, II, IO3 1 
4. Wie solt ich armer der swaere getrûwen

Conon de Bethune, Moult me semont Amors ke je m'envoise

Bertran de Born, Ai, Lemozis, francha terra cortesa

Bertran de Born, Pois als baros enoja e lur pesa

Gace Brulé, Bien ait l'amor dont l'en cuide avoir joie

Frank I, 98-107, II, 94-100 1

\section{DIETMAR VON EIST}

5. Der winter waere mir ein zît

Heinrich von Rugge, Hân ich iht vriunt, die wünschen ir

Pseudo-Veldeke, Swenn diu zît alsô gestât

Bernart de Ventadorn, Quan vei l'alauzeta mover

GTM 45 d; Gennrich, Mbd. Liedkunst 6 ?

\section{FRIEDRICH VON HUSEN}

\section{Deich von der guoten sehiet}

Bernart de Ventadorn, Pos prejatz me, senhor

Frank I, 2-5, II, 9-Io 9; zfMW vII, $92 d$; GTM $46 d$;

HGEM 20

7. Diu süezen wort hânt mir getân

Bernger von Horheim, Nu enbeiz ich doch des trankes nie

Chrestien de Troyes, D'Amors qui m'a tolu a moy

Gaucelm Faidit, Si tot m'a tarzat mon chan

Frank I, 22-27, II, 33-34 १; ZfMw vII, 96 d; GTM 49 d

8. Gelebt ich noch die lieben zît

Blondel de Nesle, Se savoient mon tourment

9. Ich denke under wîlen

Guiot de Provins, Ma joie premeraine

Vidame de Chartres, Combien que j'ai demouré

Frank I, I6-2 I, II, 87 १; GTM 47 d; Gennrich, Mbd. Liedkunst Io १;

GFML $197-98$ d

Io. Ich lobe Got der sîner güete

Gace Brulé, Pensis d'Amors voil retraire

Frank I, IO-I 5, II, 23-24 १

I I. Mîn herze den gelouben hât

Gontier de Soignies, Tant ai mon chant entrelaissié

Gontier de Soignies, Se li oisel baissent lor chans 
I 2. Mîn herze und mîn lîp diu wellent scheiden Albrecht von Johansdorf, Mich mac der tôt Conon de Bethune, Ahi, Amors, com dure departie Chastelain de Coucy, La douce vois du rossignol salvage Frank I, 28-33, Ir, 40-46 १; zfMW vII, 94 d

13. Mir ist daz herze wunt Anon., Mult m'a demoré Frank I, 6-9, II, I6-17 9; GTM 46 d

14. Si waenent sich dem tôde verzîn Anon., El dous tens que voi venir No melody

HARTMANN VON AUE

I . Ich muoz von rehte den tac iemer minnen Gace Brulé, Ire d'amors ke en mon cuer repaire Frank I, I26-30, II, II6 d, II 79

HEINRICH VON MORUNGEN

I6. Lanc bin ich geweset verdâht (Authorship?)

Anon., Je ne sui pas esbahis

Frank I, I I6-I9, II, IO9 9

17. Mirst geschên als eime kindelîne Anon., Aissi m'ave cum a l'enfant petit Frank I, I I 2-I s, no melody

\section{HEINRICH VON VELDEKE}

18. Als die vogele frewelîche

Bernart de Ventadorn, Bel m'es quant eu vei la broilla

Richart de Semilli, Quant la sesons renouvele

Richart de Semilli, De chanter m'est pris courage

19. Ich bin frô, sît uns die tage

Pierre de Molins(?), Fine amours et bone esperance

20. Sî is sô gût ende ouch so scône

Gace Brulé, Pour mal temps ne por gelee

Frank I, 34-39, no melody

21. Swer zer minne ist sô fruot

Gace Brulé, Oiés pour quoi plaing et soupir 
22. Mîn ougen wurden liebes also vol

Gaucelm Faidit, Mon cor e mi e mas bonas chansos

\section{RUDOLF VON FENIS}

23. Gewan ich ze Minnen ie guoten wân

Folquet de Marselha, Sitot me soi a tart aperceubutz

Folquet de Marselha, Tant m'abellis l'amoros pessamens

Folquet de Marselha, S'al cor plagues, ben for' oimais sazos

Frank I, 46-5 5, II, 64 १; zfMW vII, $75 d, 77 d$; GTM $48 d$

24. Minne gebiutet daz ich singe

Gace Brulé, De bone amor et de loial amie

Frank I, 56-6I, II, 66-7I १; zfMw vir, 87 d ; Gennrich, Mbd. Liedkunst 8 १

25. Mit sange wânde ich mîne sorge krenken

Ich hân mir selben gemachet die swaere

Friedrich von Husen, Si darf mich des zîhen niet

Hartwig von Rute, Mir tuot ein sorge wê in mînen muote

Bligger von Steinach, Er fünde guoten kouf an mînen jâren

Folquet de Marselha, En chantan m'aven a membrar

Folquet de Marselha, Ben ant mort mi e lor

Gaucelm Faidit, Pel messatgier que fai tan lonc estage

Gace Brulé, Merci, Amours, qu'iert il de mon martire

Gace Brulé, Tant m'ait meneit force de signoraige

Hugues de Bregi, Ausi con cil qui cuevre sa pesance

Anon., Je ne mi vueil de bone amour retraire

Anon., Espris d'amour et de longue atendance

Anon., Ce qu'Amours a si tres grande poissance

Frank I, 62-79, II, 72-84 1; zfMW VII, 89 d; GTM 4s d;

Gennrich, Mbd. Liedkunst 7 ๆ

26. Nun ist niht mêre mîn gedinge

Swer sô staeten dienest kunde

Peire Vidal, Pos tornatz sui em Proensa

Frank I, 80-8 s, II, 87 १; zfMW vII, 78-79 d ; GTM 49 d

\section{ULRICH VON GUTENBERG}

\section{Ich hôrte wol ein merlikîn singen}

Blondel de Nesle, Bien doit chanter qui fine Amors adreche Frank I, 40-45, II, 55-58 १; GTM 47 d; GFML 221-22 d 
62. CARMINA BURANA

See Aarburg, op.cit; H. Spanke, „Der Codex Buranus als Liederbuch," zfMw XIII (I93I), 24I ff.; W. Lipphardt, „Unbekannte Weisen zu den Carmina Burana," Afmw XII (I955), I 22 ff.; also 54, Walther von der Vogelweide.

Included in the Carmina Burana are the following tunes, in staffless neumes:

I. Pseudo-Dietmar, Urlop hât des sumers brehen CB I6I-I6ra

2. Heinrich von Morungen, Ich bin keiner âne krône CB I soa (f. 6Ir), incomplete

3. Reimar, Sage, daz ich dirs iemer lône CB $147 a$ (f. 6ov)

4. Pseudo-Reimar, Sold ab ich mit sorgen iemer leben св I66-I66а

5. Pseudo-Reimar, Ze niuwen fröuden stât mîn muot CB I43a (f. 59r) 


\title{
CHRONOLOGY
}

\author{
XII Century
}

45

\section{Century}

I, 3, 4, 6, 8, I0, I4, I7, I8, 20, 25, 27, 30, 36, 37, 40,4I, 43, 44, 49, $50,52,53,54,55,57$

$$
\text { XIV Century }
$$

$2,7,9,13,16,19,21,22,26,28,32,33,35,48,51,56,58,59$

$$
\text { XV Century }
$$

$15,29,31,39$

The Four Gekrönte Töne: 8-1 3, 8-4I, 9-2, 25-8, 25-9, 35-10, 35-I I See TEBM 60-6I

The Twelve Masters:

r. Herr Walter ein Landherr, von der Fogel weid

2. Wolffgang Rohn ein Ritter

3. Conrat Marner ein Edelman

4. D: Henrich Frauenlob zu Menz

5. D: Henrich Mugeling D: teologie

6. M: Klingesohr

7. M: Starcke Popp

8. Bartel Regenbogen, ein Schmid

9. Der Römer war von Zwickau, ein burger

Io. Der Kanzeler war ein fischer

I I. Conrad von Wirzberg

I 2. Der allte Stoll

List from: Adam Puschmann, Gruntlicher Bericbt des deutschen Meister Gesanges, 1584 




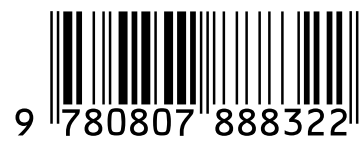

